\title{
Influence of Brain Gangliosides on the Formation and Properties of Supported Lipid Bilayers
}

\author{
Luke Jordan, Megan Blauch, Ashley Baxter, Jennie Cawley, Nathan Wittenberg
}

Submitted date: 21/06/2019 - Posted date: 24/06/2019

Licence: CC BY-NC-ND 4.0

Citation information: Jordan, Luke; Blauch, Megan; Baxter, Ashley; Cawley, Jennie; Wittenberg, Nathan (2018): Influence of Brain Gangliosides on the Formation and Properties of Supported Lipid Bilayers. ChemRxiv. Preprint.

This is a comprehensive study of the effects of the four major brain gangliosides (GM1, GD1b, GD1a, and GT1b) on the adsorption and rupture of phospholipid vesicles on $\mathrm{SiO} 2$ surfaces for the formation of supported lipid bilayer (SLB) membranes. Using quartz crystal microbalance with dissipation monitoring (QCM-D) we show that gangliosides GD1a and GT1b significantly slow the SLB formation process, whereas GM1 and GD1b have smaller effects. This is likely due to the net ganglioside charge as well as the positions of acidic sugar groups on ganglioside glycan head groups. Data is included that shows calcium can accelerate the formation of ganglioside-rich SLBs. Using fluorescence recovery after photobleaching (FRAP) we also show that the presence of gangliosides significantly reduces lipid diffusion coefficients in SLBs in a concentration-dependent manner. Finally, using QCM-D and GD1a-rich SLB membranes we measure the binding kinetics of an anti-GD1a antibody that has similarities to a monoclonal antibody that is a hallmark of a variant of Guillain-Barre syndrome.

File list (2)

Jordan_chemRxiv_v2.pdf (1.20 MiB)

view on ChemRxiv - download file

Jordan_chemRxiv_SI_v2.pdf (2.07 MiB)

view on ChemRxiv - download file 


\section{Influence of Brain Gangliosides on the Formation and Properties of Supported Lipid Bilayers}

Luke R. Jordan, ${ }^{a}$ Megan E. Blauch, ${ }^{a, b}$ Ashley M. Baxter, ${ }^{a, b}$ Jennie L. Cawley, ${ }^{a, b}$ and Nathan J. Wittenberg $^{a *}$

a Department of Chemistry, Lehigh University, Bethlehem, Pennsylvania, 18015, U.S.A.

${ }^{\mathrm{b}}$ These authors contributed equally

*Corresponding author. Email address: njw@lehigh.edu

KEYWORDS: ganglioside, glycolipid, supported lipid bilayer, membrane, quartz crystal microbalance 


\section{ABSTRACT}

Gangliosides are glycolipids that are enriched on the outer surface of cell membranes. Gangliosides are receptors for a number of signaling molecules and toxins, and therefore are often incorporated into biosensors. Many of these biosensors incorporate gangliosides into supported lipid bilayers which are formed by the spontaneous rupture of unilamellar vesicles on glass or $\mathrm{SiO}_{2}$ substrates. In this work we used quartz crystal microbalance with dissipation monitoring (QCMD) to investigate how the presence of the four major brain gangliosides (GM1, GD1a, GD1b, and GT1b) influences the process of supported lipid bilayer formation on $\mathrm{SiO}_{2}$ surfaces. We show that the rate of supported bilayer formation is dependent on both the charge and position of sialic acid moieties on ganglioside molecules. Additionally, $\mathrm{Ca}^{2+}$ can accelerate ganglioside-rich supported bilayer formation, but the degree of acceleration differs for vesicles containing different gangliosides. Fluorescence recovery after photobleaching measurements show that the presence of all gangliosides reduces lipid diffusion coefficients in a concentration-dependent manner, and that $\mathrm{Ca}^{2+}$ slows lipid diffusion in membranes with and without gangliosides. Finally, we use ganglioside-rich supported bilayers to measure binding constants for a GD1a-binding antibody that has similar properties to antibodies present in a variant of Guillain-Barré syndrome. 


\section{INTRODUCTION}

The cellular plasma membrane is a complex environment composed of a wide variety of lipids, embedded with proteins, and decorated with carbohydrates. Lipid-carbohydrate conjugates (glycolipids) play essential roles in cellular recognition, adhesion, and infection. One family of glycolipids, gangliosides, are found in particularly high abundance in the vertebrate nervous system.[1] Gangliosides contain one or more sialic acid residues which are deprotonated at physiological $\mathrm{pH}$. The predominant sialic acid found on human gangliosides is $\mathrm{N}$-acetylneuraminic acid.[2] The most common gangliosides in the nervous system are GM1, GD1a, GD1b, and GT1b. Together, these four gangliosides account for roughly $94 \%$ of the gangliosides found in the human brain.[3] Gangliosides are enriched on the extracellular membrane leaflet and are known to cluster with cholesterol in membrane microdomains $[4,5]$ and cluster with themselves in the absence of cholesterol.[6]

In cells and tissues, gangliosides interact with a number of different molecules. For example, GD1a and GT1b on axons interact with Siglec-4, also called myelin-associated glycoprotein (MAG), in the periaxonal space between an axon and the first wrap of myelin.[7] Gangliosidebinding antibodies are also a hallmark of Guillain-Barré syndrome.[8] In addition to endogenous interactions, gangliosides can function as receptors for a number of toxins and viruses.[9] Perhaps the best known example is cholera toxin, in which the cholera toxin B subunit (CTB) binds GM1 with high affinity.[10] Gangliosides are also receptors for heat labile enterotoxin,[11] tetanus toxin,[12] and botulinum toxin.[12] Gangliosides also interact with pathogenic fibrils of $\beta$-amyloid [13] and $\alpha$-synuclein.[14]

Because gangliosides bind important endogenous molecules, toxins, and viruses, a number of sensing strategies have been devised to quantitatively probe these interactions. Many approaches 
use model membrane systems, like giant unilamellar vesicles (GUVs), liposomes, or supported lipid bilayers (SLBs). The lipid composition of these model membranes can be precisely controlled, and the membranes can be incorporated into a number of sensor architectures with relative ease.[15-17] A variety of fluorescence assays have been used to investigate binding between glycolipids or gangliosides in model membranes and toxins or virus particles.[18-22] Ganglioside binding can also be detected with label-free methods like backscattering interferometry,[23] colloidal bead assembly,[24] surface plasmon resonance (SPR), $[10,25,26]$ localized surface plasmon resonance,[27] isothermal titration calorimetry,[28] and quartz crystal microbalance with dissipation monitoring (QCM-D).[29, 30]

Many strategies for characterizing ganglioside interactions rely upon the creation of gangliosiderich SLBs. Formation of SLBs can be accomplished by the spontaneous rupture of vesicles,[31] Langmuir-Blodgett methods,[32] or other techniques.[33, 34] It is well established that a number of factors, including the substrate chemistry and the lipid composition of precursor vesicles, can influence SLB formation via vesicle rupture.[35] While the influence of GM1 on SLB formation by vesicle rupture has been examined,[30] there are few studies on the effects of the more highlycharged GD1a, GD1b, and GT1b on SLB formation. In this work we examine the influence of the four major brain gangliosides on the formation of SLBs by vesicle rupture. We show that not only the ganglioside charge but also the position of sialic acid moieties, has a significant influence on the transition from adsorbed vesicles to a SLB. We also find that the presence of $\mathrm{Ca}^{2+}$ can significantly accelerate vesicle rupture kinetics. Fluorescence recovery after photobleaching (FRAP) studies show that increasing ganglioside concentrations reduces lipid diffusion coefficients significantly, and the presence of $\mathrm{Ca}^{2+}$ slows lipid diffusion in SLBs with and without 
gangliosides. Finally, we use membranes rich in GD1a to examine antibody binding kinetics with QCM-D.

\section{RESULTS AND DISCUSSION}

\section{Formation of SLBs containing brain gangliosides}

The four brain gangliosides examined in these studies are GM1, GD1a, GD1b, and GT1b. The structures, both in schematic illustrations as well as molecular models created with the glycolipid modeler in CHARMM-GUI,[36] are shown in Fig. 1. The four gangliosides have head groups with a common glycan backbone consisting of glucose, galactose, and $N$-acetylgalactosamine (GalNAc).[37] Where they differ, however, is in the number and linkages of sialic acid moieties. At physiological $\mathrm{pH}$ the sialic acid groups confer a net negative charge on gangliosides, with the charge equal to the number of sialic acids per molecule. Thus GM1, GD1a, GD1b, and GT1b have nominal charges of $-1,-2,-2$, and -3 , respectively. Simulations[38] and atomic force microscopy (AFM) measurements on lipid monolayers[39] and SLBs[6] have shown that the glycan head groups of gangliosides extend between $0.7-2.0 \mathrm{~nm}$ above the head groups of the background phospholipids. This makes it likely that during the vesicle adsorption process, the glycan head groups are the first entities on vesicles that encounter the substrate.

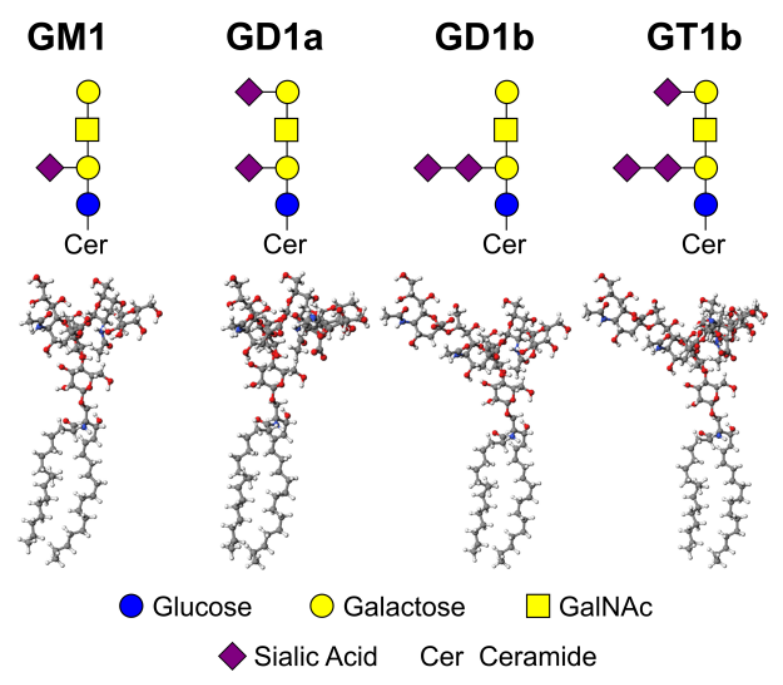


Figure 1. Structures of GM1, GD1a, GD1b, and GT1b gangliosides. (Top) Schematic illustrations of ganglioside structures. (Middle) Ball-and-stick models of ganglioside structures. (Bottom) Legend for the illustrated structures.

As precursors to the SLBs, we formed vesicles composed of dioleoylphosphatidylcholine (DOPC) and $1-5 \mathrm{~mol} \%$ gangliosides by hydration in Tris buffer, bath sonication, and extrusion through 50-nm pore size filters. To monitor the formation of $\mathrm{SLBs}_{\text {on }} \mathrm{SiO}_{2}$ surfaces, we employed QCM-D. Using QCM-D, it is possible to monitor the adsorption of vesicles to surfaces by observing negative shifts in the resonant frequency $(\Delta F)$ of the QCM-D sensor, which correspond to mass added to the surface.[40] As the vesicles adsorb to the surface, the dissipation signal $(\triangle D)$ also increases due to the viscoelasticity of a layer of intact vesicles.[41-43] For the case of zwitterionic vesicles on a $\mathrm{SiO}_{2}$ surface, a critical population of vesicles must be adsorbed to the surface before the vesicle rupture cascade can begin.[44] Once vesicles begin to rupture, the frequency shift reaches a minimum, then shifts back toward more positive values as aqueous solution from the interior of vesicles is liberated. The dissipation signal also exhibits a maximum as the viscoelastic adsorbed vesicle layer begins the transition into a rigid SLB film.[41] The time required to reach the minimum in the frequency curve is $t_{c r i t}$, and the magnitude of frequency shifts at this time is $\Delta F_{c r i t}$. The value of $\Delta D_{\text {crit }}$ was taken from the maximum in the dissipation curve. Once the vesicle rupture process is complete and a SLB is formed, the $\Delta F$ and $\Delta D$ signals reach stable plateaus, which are referred to as $\Delta F_{S L B}$ and $\triangle D_{S L B}$, respectively. The critical and SLB values are illustrated in the annotated QCM-D curves in Fig. 2a. Values of $t_{\text {crit }}, \Delta F_{\text {crit }}$, and $\Delta D_{\text {crit }}$ can depend on a number of factors, including vesicle size, vesicle charge, vesicle infusion rate, vesicle concentration, buffer ionic strength, buffer $\mathrm{pH}$, presence of divalent cations, and temperature.[35, 
$42,45-54]$ The values of $\Delta F_{\text {crit }}, \Delta D_{\text {crit }}, \Delta F_{S L B}$, and $\Delta D_{S L B}$ we measured for DOPC SLB formation agree with previous reports.[42, 55]
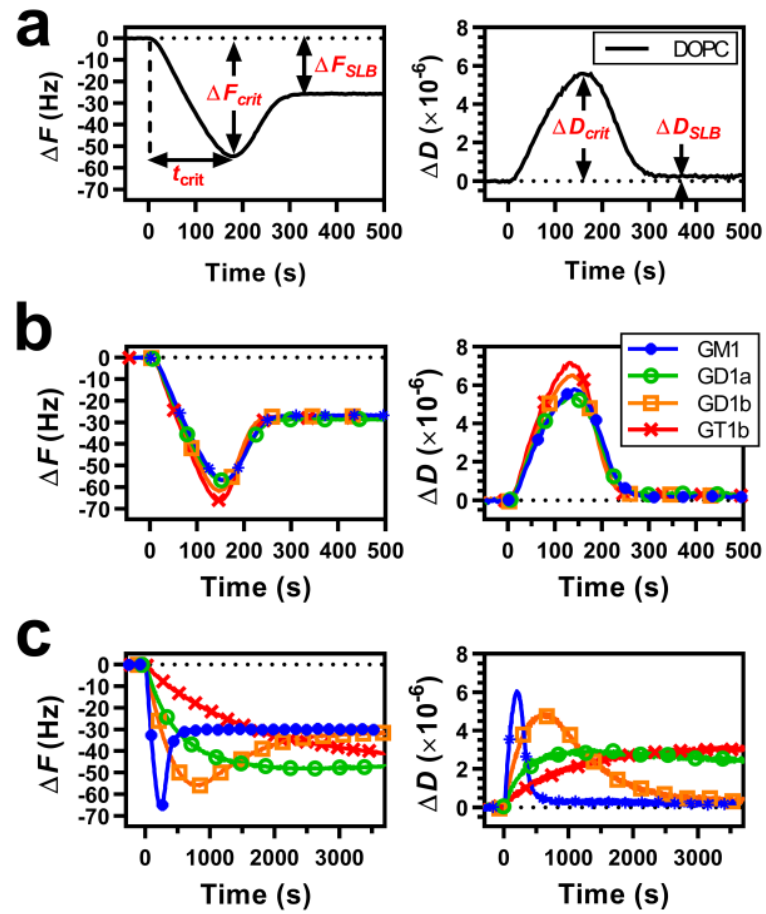

Figure 2. Formation of ganglioside-rich SLBs monitored with QCM-D. (a) Frequency (left) and dissipation (right) shifts for adsorption and rupture of DOPC vesicles. The annotations are defined in the text. (b) Frequency (left) and dissipation (right) shifts for adsorption and rupture of DOPC vesicles containing 1\% GM1, GD1a, GD1b, or GT1b. (c) Frequency (left) and dissipation (right) shifts for adsorption and rupture of DOPC vesicles containing 5\% GM1, GD1a, GD1b, or GT1b.

Next, we evaluated the influence of increasing mole fractions of GM1, GD1a, GD1b, and GT1b on the adsorption and rupture of DOPC vesicles in Tris buffer. Vesicles were prepared containing $1,2,3,4$, or $5 \%$ of the gangliosides and were injected into the QCM-D. Figures $2 \mathrm{~b}$-c show frequency and dissipation curves for vesicles containing either 1 or $5 \%$ of the gangliosides. Frequency and dissipation curves from 2, 3, and 4\% ganglioside vesicles are shown in Fig. S1. Fig. $2 \mathrm{~b}$ shows curves for the rupture of $1 \%$ ganglioside vesicles which are not statistically different than the pure DOPC control. The effects of gangliosides on SLB formation become significant at 
higher mole fractions (Fig. 2c). Vesicles containing 5\% GM1 or GD1b form SLBs via a two-step adsorption-rupture pathway. The $\Delta F$ and $\Delta D$ signals indicate that vesicles containing $5 \%$ GD1a or GT1b do not rupture to form complete SLBs. While Fig. 2c only shows the first hour (3600 s) of the QCM-D recordings, the samples with 5\% GD1 a and GT1b were exposed to the QCM-D sensor for up to 7.5 hours, but we did not observe a frequency or dissipation plateau to indicate formation of a complete SLB (Fig. S2).

A summary of all figures of merit ( $\Delta F_{c r i t}, \Delta D_{\text {crit }}, t_{c r i t}$, and $\left.\Delta F_{S L B}\right)$ is shown in Fig. 3. In general, as the concentration of gangliosides in the vesicles increases, the values of $\Delta F_{c r i t}$ increase. This indicates that as the ganglioside content increases, more vesicles need to adsorb to the surface before the rupture cascade begins. This could stem from weaker adhesion of vesicles to the $\mathrm{SiO}_{2}$ substrate. Negative surface charges on both the $\mathrm{SiO}_{2}$ and the ganglioside-containing vesicles results in electrostatic repulsion that counteracts attractive van der Waals interactions between the vesicles and the $\mathrm{SiO}_{2}$.[50] Furthermore, increasing the concentration of gangliosides increases the amount of hydrogen bonding between water and the vesicle surface, and thus increases the hydration repulsive force.[56] Because hydration repulsive forces and electrostatic repulsion oppose adhesion, vesicles with higher ganglioside content will have lower adhesion energies. For a constant membrane bending modulus, lowering the adhesion energy reduces vesicle deformation by decreasing the size of the membrane-substrate contact patch.[57] Thus, for the rupture cascade to begin, the vesicles need to pack more tightly, which induces stresses on the vesicle membranes that can initiate their rupture.[58] The vesicles with 5\% GD1a and GT1b exhibit a $\Delta F_{\text {crit }}$ value that is less than the $\Delta F_{\text {crit }}$ of vesicles with $4 \%$ GD1a and GT1b. Critical values were taken from the minimum point in the frequency curves. In the case of 5\% GD1a and GT1b vesicles, a minimum 
is observed; however the frequency never reaches a stable plateau (Fig. S2). This suggests that $5 \%$ GD1a and GT1b vesicles transition from an intact adsorbed state to a SLB very slowly.

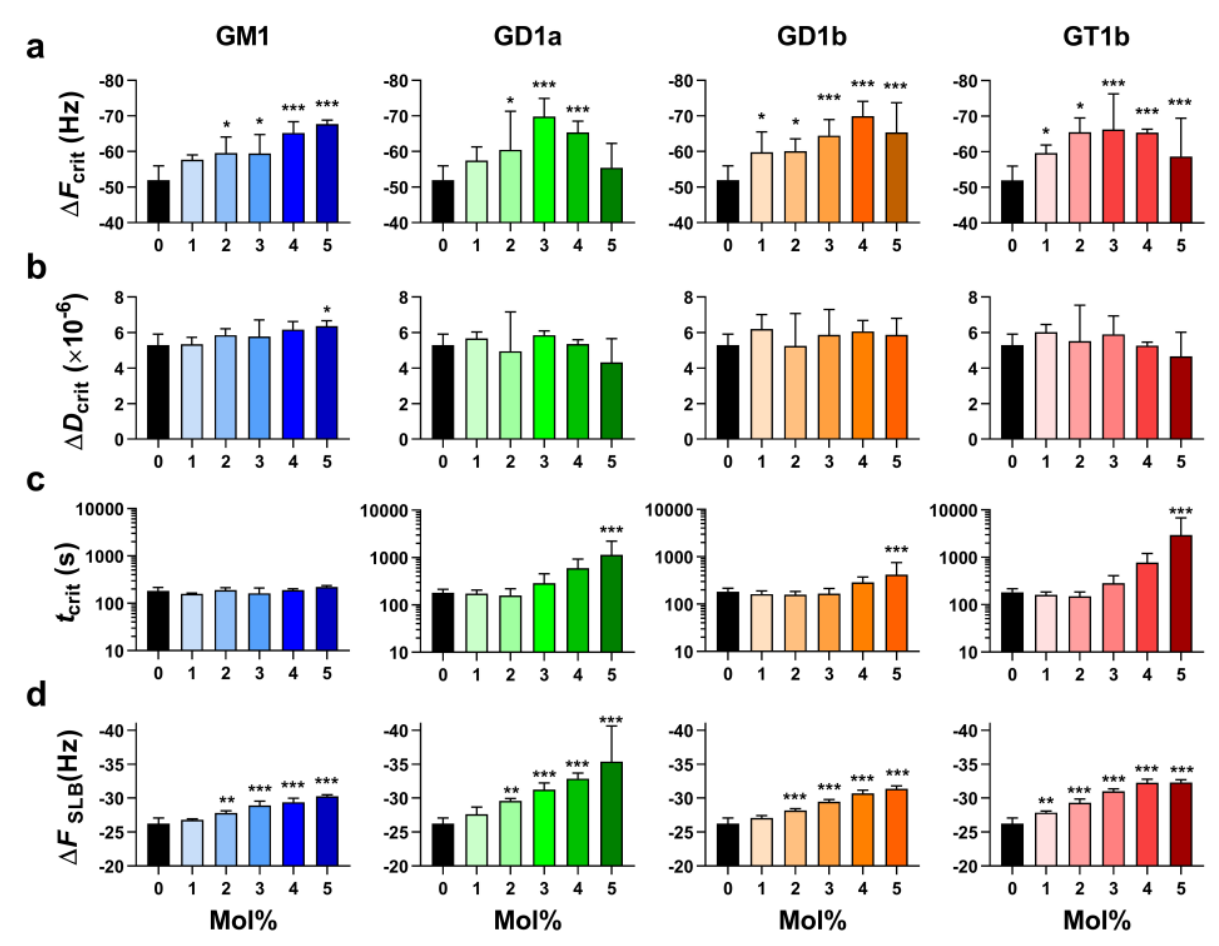

Figure 3. Influence of ganglioside type and concentration on vesicle adsorption and rupture. (a) $\Delta F_{c r i t}$ values as a function of ganglioside type and concentration. (b) $\Delta D_{\text {crit }}$ values as a function of ganglioside type and concentration. (c) $t_{c r i t}$ values as a function of ganglioside type and concentration. (d) $\Delta F_{S L B}$ values as a function of ganglioside type and concentration. ${ }^{*} p<0.05,{ }^{* *} p$ $<0.01,{ }^{* * *} p<0.001$ compared to vesicles composed solely of DOPC.

While the critical frequencies increase as ganglioside content increases, the critical dissipation values ( $\Delta D_{\text {crit }}$ ) across all gangliosides at all concentrations, with the exception of $5 \% \mathrm{GM} 1$, do not significantly differ from DOPC controls (Fig. $3 \mathrm{~b}$ ). The $t_{c r i t}$ is significantly different from DOPC controls for 5\% GD1a, GD1b, and GT1b (Fig. 3c). When comparing tcrit for GD1a, GD1b, and GT1b, a dependence on ganglioside charge and structure emerges. The $t_{c r i t}$ values for 5\% GD1a and GT1b are much larger than those of 5\% GD1b. Among the gangliosides studied here, GT1b has the largest negative charge (-3). It is likely that vesicles with GT1b take the longest to reach 
the critical point for vesicle rupture due to increased electrostatic and hydration repulsive forces between the vesicles and the $\mathrm{SiO}_{2}$ surface. Increased membrane-membrane repulsion between surface-adsorbed vesicles also contribute to the increase in $t_{\text {crit }}$. Increased repulsion between the vesicles and the surface reduces the degree of vesicle deformation arising from adhesive interactions that stress vesicles, which ultimately drive vesicle rupture. Analogous results were obtained by Richter et al. when examining the rupture of phosphatidylserine (PS)-rich vesicles on $\mathrm{SiO}_{2}$. PS lipids have a -1 charge, and when the PS content was increased from zero to $33 \%$, there was more than a 20 -fold increase in the time required to form a SLB.[42] However, at $20 \%$ PS, there was smaller increase of $50 \%$ in the time required for SLB formation.

Vesicles containing $5 \%$ GD1a have a $t_{\text {crit }}$ that is nearly twice that of $5 \%$ GD1b vesicles. GD1a and GD1b both contain two sialic acid groups, and therefore both have a charge of -2 . However, the position of sialic acid linkages differ between these two molecules. GD1b has two sialic acids linked to the internal galactose, while GD1a has one sialic acid linked to the internal galactose and one linked to the terminal galactose (Fig. 1). This structural difference may alter vesicle-substrate and vesicle-vesicle interactions for vesicles containing these gangliosides. Though GD1b has two sialic acid groups, one of them is somewhat hidden and could cause steric screening. A compounding effect is the sialic acid on the terminal galactose of GD1a, which is more likely to have direct interactions with both the substrate and terminal sialic acids on GD1a molecules on neighboring vesicles. GT1b also has a sialic acid on its terminal galactose, which may further explain the retarding effect it has on vesicle rupture. To isolate the effects of sialic acid moieties on the terminal galactose of gangliosides, one would need to investigate the adsorption and rupture of vesicles containing GM1b or GD1c. However, these gangliosides are either expressed 
transiently during brain development or are present only in trace amounts in the developed mammalian brain,[59] and thus are quite difficult to isolate in appreciable quantities.

The final frequency shift corresponding to the formation of a SLB $\left(\Delta F_{S L B}\right)$ increases as the concentration of gangliosides in the vesicles increases (Fig. 3d). The measured $\Delta F_{S L B}$ value is proportional to the product of the SLB density and its thickness,[40] and the increase $\Delta F_{S L B}$ occurs due to increases in both density and thickness. The presence of gangliosides has a condensing effect on the SLB, [30], $[60,61]$ which increases the density of the SLB. Additionally, the glycan head groups of the gangliosides increase the SLB thickness.

\section{Effect of $\mathrm{Ca}^{2+}$ on SLB formation}

Divalent cations, such as $\mathrm{Ca}^{2+}$ and $\mathrm{Mg}^{2+}$, have been shown to accelerate the rate of vesicle rupture on $\mathrm{SiO}_{2} \cdot[54,62] \mathrm{Ca}^{2+}$ can also accelerate the rupture of negatively charged lipid vesicles to form $\mathrm{SLBs}_{\text {on }} \mathrm{SiO}_{2}$ and $\mathrm{TiO}_{2}$ surfaces.[42, 63] $\mathrm{Ca}^{2+}$ binds lipid phosphates and carbonyls, which induces a slight positive charge to the membrane. This strengthens adhesion between vesicles and negatively charged surfaces. To demonstrate the effect of $\mathrm{Ca}^{2+}$ on the formation of gangliosiderich SLBs, we prepared vesicles composed of DOPC and $1-5 \%$ of each of the brain gangliosides as described above. Then, using QCM-D, we observed their adsorption and rupture in the presence of $2 \mathrm{mM} \mathrm{Ca}^{2+}$. Frequency and dissipation responses are for $5 \%$ ganglioside vesicles are shown in Fig. 4a-b, respectively. Fig. 4c compares the $t_{\text {crit }}$ for all ganglioside concentrations in the absence and presence of $\mathrm{Ca}^{2+}$. For singly-charged GM1, $\mathrm{Ca}^{2+}$ makes little difference in $t_{\text {crit. }}$. However, for GD1a, GD1b, and GT1b, the presence of $\mathrm{Ca}^{2+}$ has a pronounced acceleration effect. Vesicles with $5 \%$ GD1b and GT1b are the most susceptible to the presence of $\mathrm{Ca}^{2+}$; their $t_{\text {crit }}$ values decrease roughly 4- and 10-fold, respectively. Vesicles with GD1a also reach $t_{c r i t}$ faster in the presence of $\mathrm{Ca}^{2+}$, though the effect is more moderate (Fig. $4 \mathrm{c}$ ). Even with $\mathrm{Ca}^{2+}$ present the $\Delta F_{S L B}$ value for $5 \%$ 
GD1a does not indicate the presence of a complete SLB. Rather, the final configuration is a combination of intact vesicles and SLB patches, similar to when $\mathrm{Ca}^{2+}$ is absent. Alternative approaches could be taken to form complete SLBs with high concentrations of GD1a. For example, solvent-assisted lipid bilayer formation[64] has been used to make SLBs on substrates where spontaneous rupture does not occur,[33] or with lipid compositions that are not susceptible to spontaneous rupture.[65]

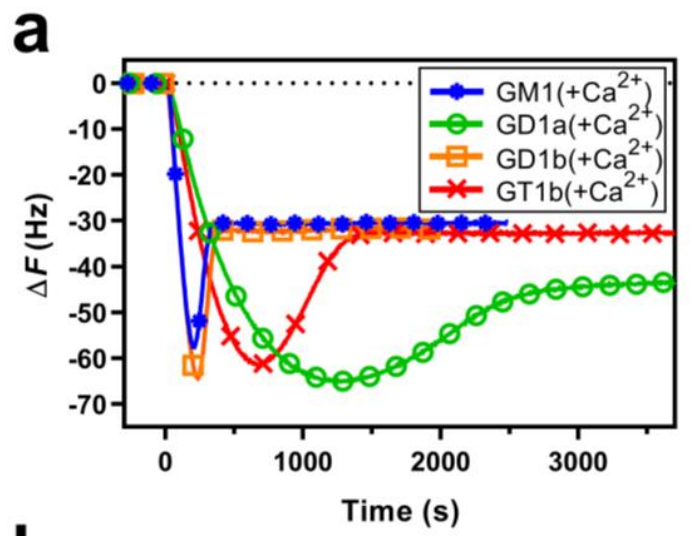

b
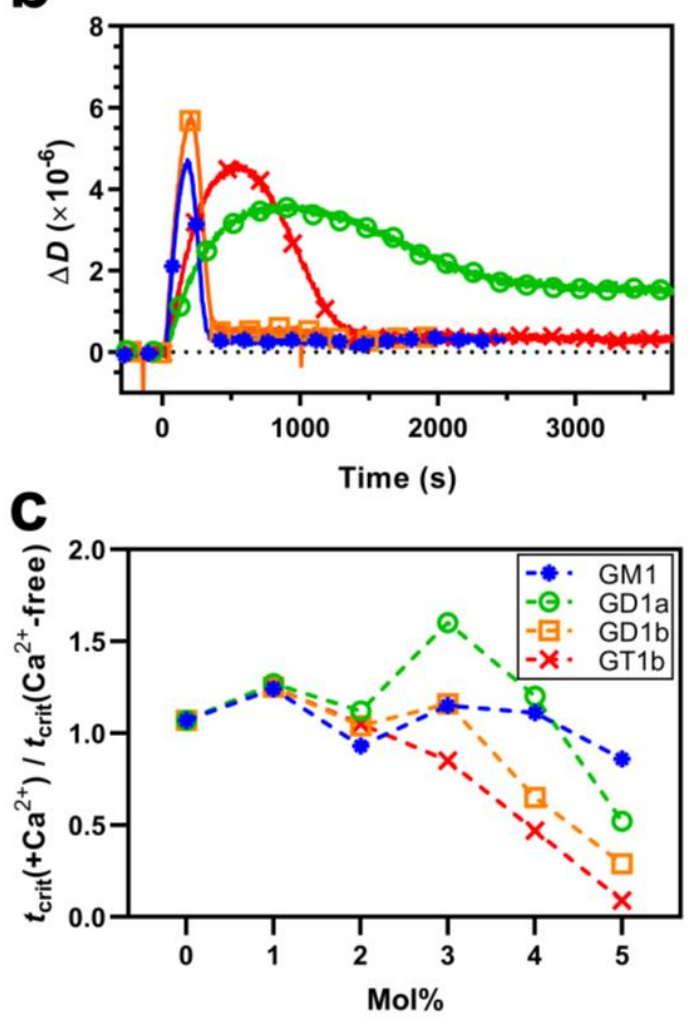
Figure 4. Effects of $\mathrm{Ca}^{2+}$ on the formation of ganglioside-rich SLBs. QCM-D measurements of vesicles with $5 \%$ gangliosides: (a) $\Delta F$ and (b) $\Delta D$. (c) Ratio of $t_{\text {crit }}\left(+\mathrm{Ca}^{2+}\right) / t_{\text {crit }}\left(\mathrm{Ca}^{2+}\right.$-free) across all concentrations and different gangliosides.

\section{Influence of gangliosides and $\mathrm{Ca}^{2+}$ on lipid diffusion}

Lipid diffusion coefficients in SLBs were examined with fluorescence recovery after photobleaching (FRAP). In these experiments, SLBs containing DOPC, varying amounts of gangliosides, and $0.1 \%$ dihexanoylphosphatidylethanolamine-Texas Red (TR-DHPE) were formed on glass coverslips by vesicle rupture in Tris buffer with $2 \mathrm{mM} \mathrm{Ca}^{2+}$. After photobleaching a small circular area $(\sim 10 \mu \mathrm{m}$ diameter $)$ of the SLB, fluorescence recovery was recorded for $2 \mathrm{~min}$. The SLBs were then washed with Tris buffer containing EDTA to chelate $\mathrm{Ca}^{2+}$, and a second FRAP measurement was made after the subsequent washout of EDTA with $\mathrm{Ca}^{2+}$-free Tris buffer. Fluorescence recovery before and after $\mathrm{Ca}^{2+}$ chelation was analyzed to calculate the lipid diffusion coefficient using the Hankel transform method described by Jönsson et al.[66] The FRAP results are summarized in Fig. 5 and in Table S1. FRAP images are shown in Fig. S3-S7.

We measured diffusion coefficients of $2.30 \pm 0.22 \mu \mathrm{m}^{2} / \mathrm{s}$ and $2.75 \pm 0.26 \mu \mathrm{m}^{2} / \mathrm{s}$ for a $100 \%$ DOPC SLB before and after chelation of $\mathrm{Ca}^{2+}$, respectively. These values are within the range of values typically reported for fluid PC bilayers on solid supports.[66, 67] Prior to addition of EDTA, the lipid diffusion coefficients in SLBs tend to decrease as the concentration of gangliosides increases. The same trend is observed after $\mathrm{Ca}^{2+}$ chelation with EDTA. Upon $\mathrm{Ca}^{2+}$ chelation with EDTA, the diffusion coefficients for a given SLB composition generally increased. The increases the diffusion coefficients are, for the most part, statistically significant (Table S1). Thus our FRAP results show that gangliosides reduce lipid diffusion coefficients in the presence and absence of $\mathrm{Ca}^{2+}$. Furthermore, the presence of $\mathrm{Ca}^{2+}$ causes an overall reduction in lipid diffusion coefficients. The 5\% GD1a sample, which exhibited signs of intact vesicles and supported bilayer patches in 
the QCM-D experiments with $\mathrm{Ca}^{2+}$ (Fig. 4a,b), showed complete SLBs formation in the fluorescence microscopy experiments (Fig. S5). This may reflect subtle differences in the preparation of the glass coverslips used for FRAP and the $\mathrm{SiO}_{2}$ surfaces used for QCM-D.

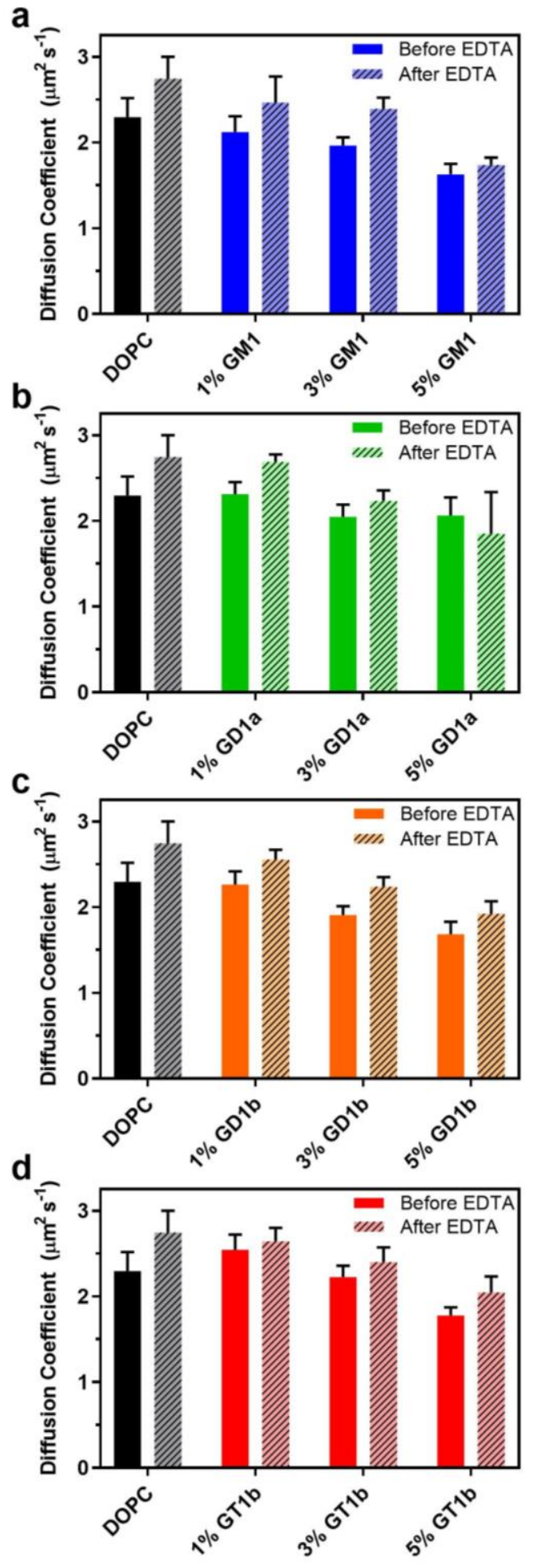


Figure 5. Influence of ganglioside concentration and $\mathrm{Ca}^{2+}$ on lipid diffusion coefficients in SLBs. Diffusion coefficients were measured with FRAP before (solid bars) and after (hatched bars) $\mathrm{Ca}^{2+}$ chelation with EDTA and subsequent washout.

Other groups have also observed ganglioside-dependent reduction in lipid diffusion coefficients. For example, Weng et al. observed $9 \%$ and $16 \%$ reduction in diffusion coefficients for TR-DHPE in egg PC SLBs containing 2\% and 5\% GM1, respectively.[30] Using POPC SLBs, Sagle et al. observed a $24 \%$ reduction in lipid diffusion coefficient when the GM1 concentration was raised from $0 \%$ to $8 \%$.[68] We observed reductions in diffusion coefficients of $8 \%$ and $29 \%$ for $1 \%$ and $5 \%$ GM1, respectively. The reduction of diffusion coefficients could be due to formation of ganglioside clusters, which have been observed in SLBs in the absence of cholesterol. GM1 clusters in POPC SLBs were measured to be roughly 7 to $20 \mathrm{~nm}$ in diameter as the concentration of GM1 is increased from 0.1 to $10 \%$.[6] Such clusters could act as obstacles to TR-DHPE diffusion, which would lower the observed diffusion coefficients. Ganglioside clustering can be driven by a combination of hydrogen bonding interactions between glycan head groups[69] and ganglioside tail group interactions. Ganglioside tails are composed of ceramide that is made up of sphingosine and a fatty acid. The bovine ganglioside ceramide tails are primarily composed of a saturated (18:0) fatty acid chain and an unsaturated (18:1) sphingosine chain. [70] By itself as little as $2 \%$ ceramide is able to induce a significant increase in lipid order in PC membranes.[71] Thus it is likely that the reductions in diffusion coefficients we observed are due to ganglioside cluster formation driven by hydrogen bonding between glycan heads along with ceramide-ceramide interactions.

$\mathrm{Ca}^{2+}$ ions bind phospholipids and gangliosides in membrane bilayers.[72, 73] Our results show that $\mathrm{Ca}^{2+}$ chelation increases lipid diffusion coefficients for membranes composed solely of DOPC as well as those containing gangliosides. Spectroscopic and molecular dynamics simulations have 
shown that $\mathrm{Ca}^{2+}$-lipid interactions cause an increase in lipid packing density and tail group order, $[74,75]$ which explains why lower diffusion coefficients are observed when $\mathrm{Ca}^{2+}$ is present. Stronger membrane-substrate interactions in the presence of $\mathrm{Ca}^{2+}$ can also contribute to reduction in lipid diffusion coefficients.

\section{Measuring antibody binding constants using ganglioside-rich SLBs}

We used QCM-D to investigate the binding between a monoclonal IgG anti-GD1a antibody and GD1a embedded in SLBs. Abnormally high levels of anti-GM1 and anti-GD1a antibodies are found in patients with the acute motor axon neuropathy (AMAN) variant of Guillain-Barré syndrome, which can result in degeneration of motor neurons.[8] The anti-GD1a antibody used here (GD1a-1) binds motor neurons rather than sensory neurons, despite the two cell types having similar levels of GD1a.[76, 77] Additionally, GD1a-1 displays similar tissue binding patterns as serum antibodies from AMAN patients.[78] To investigate how GD1a-1 interacts with GD1a in model membranes, we functionalized a $\mathrm{SiO}_{2}$-coated QCM-D sensor with a SLB containing $1 \%$ GD1a. The SLB was formed by rupture of vesicles in Tris buffer containing $2 \mathrm{mM} \mathrm{Ca}^{2+}$ (Fig. S8). Then as a negative control, $10 \mathrm{nM}$ cholera toxin B-subunit (CTB) was injected over the SLB. The negligible frequency shift of the QCM-D indicates that CTB does not bind this lipid composition (Fig. 6). Additional SLBs containing 1\% GD1a were exposed to 1,5 , or $50 \mathrm{nM}$ GD1a-1 and binding was monitored with QCM-D. As expected, the maximum frequency shift increased with increasing GD1a-1 concentration (Fig. 6). To determine the rate constants of association $\left(k_{a}\right)$ and dissociation $\left(k_{d}\right)$, the antibody binding curves were fit to exponential models for association and dissociation (Fig. 6). The ratio of the rate constants $\left(k_{d} / k_{a}\right)$ gives the equilibrium dissociation constant $K_{D}$. For GD1a-1 binding to $1 \%$ GD1a in a SLB, we determined a $K_{D}$ of $2.4 \pm 1.3 \mathrm{nM}$. 
Monoclonal $\mathrm{IgG}$ antibodies typically display $K_{D}$ values over a wide range, from low $\mathrm{pM}$ to $\mathrm{nM}$. Thus our measured $K_{D}$ for the monoclonal GD1a-1 is within the expected range.

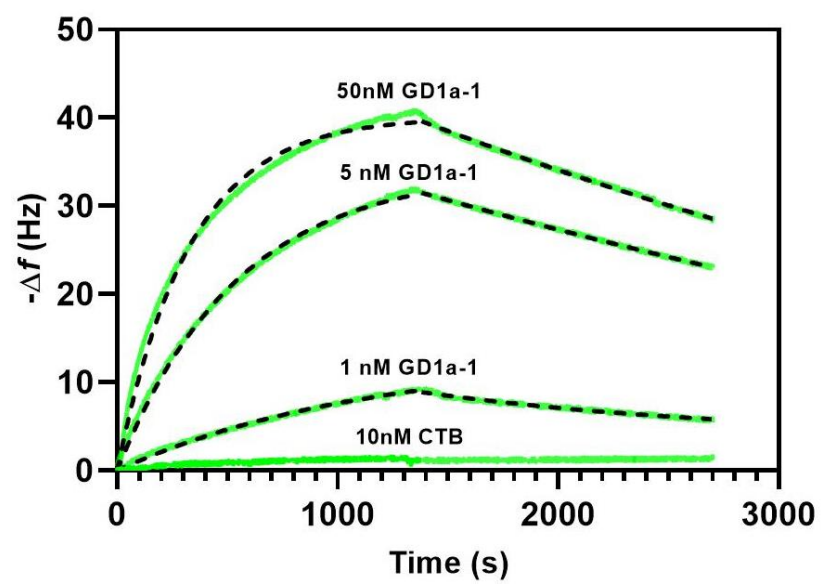

Figure 6. QCM-D frequency shift curves showing 1, 5, and $50 \mathrm{nM}$ GD1a-1 antibody binding to SLBs with 1\% GD1a. The experimental data is represented with solid green lines, and the fits are shown with dashed black lines. A curve showing $10 \mathrm{nM}$ CTB does not bind to a $1 \%$ GD1a SLB is included as a negative control.

We conducted additional QCM-D binding assays and controls using GM1-rich SLBs. In these experiments, a SLB composed of DOPC and 5\% GM1 was formed by vesicle rupture in the presence of $2 \mathrm{mM} \mathrm{Ca}^{2+}$ in Tris buffer. Next we injected $10 \mathrm{nM} \mathrm{GD1a-1,} \mathrm{which} \mathrm{did} \mathrm{not} \mathrm{bind} \mathrm{the}$ GM1-rich SLB, indicating antibody specificity. Subsequent injections of escalating concentrations of CTB resulted in increasing shifts in the frequency signal (Fig. S9). During the wash steps of our binding assay, the amount of CTB dissociation was negligible, which is expected due to the very high affinity CTB has for GM1.[10,25]

\section{SUMMARY AND CONCLUSIONS}

In summary, the presence of gangliosides in vesicles can significantly alter the process of SLB formation by spontaneous vesicle rupture. The gangliosides GD1a and GT1b hinder the SLB formation process much more than GM1 and GD1b. This is likely due to the presence of sialic acids on the terminal galactose of the glycan head group. Electrostatic repulsion between the $\mathrm{SiO}_{2}$ 
or glass substrates and the terminal sialic acid may account for the relatively slow vesicle rupture kinetics. The inclusion of $\mathrm{Ca}^{2+}$ in buffers has been previously shown to accelerate vesicle rupture, especially for lipid compositions containing anionic lipids. We observe similar effects, with $\mathrm{Ca}^{2+}$ resulting in up to a 10 -fold reduction in the critical time for the vesicle rupture cascade to begin when gangliosides are present at the 5\% level. Lipid diffusion coefficients tend to decrease as ganglioside concentrations in SLBs increase. This trend is observed in the presence of $\mathrm{Ca}^{2+}$ and after $\mathrm{Ca}^{2+}$ chelation. Chelation and removal of $\mathrm{Ca}^{2+}$ significantly increases lipid diffusion coefficients for most compositions studied. Finally, we used a QCM-D sensor functionalized with SLBs containing GD1a to measure the binding kinetics and affinity of GD1a-1, a monoclonal antibody with properties similar to those found in the AMAN variant of Guillain-Barré syndrome. This antibody binds GD1a with a $K_{D}$ of $2.4 \mathrm{nM}$.

\section{MATERIALS AND METHODS}

\section{Reagents and Chemicals}

Lipids were purchased and stored in chloroform or a chloroform/methanol mixture. All lipid stock vials were backfilled with Ar gas and stored in a -20 ${ }^{\circ} \mathrm{C}$ freezer. 1,2-dioleoyl-sn-glycero-3phosphocholine (DOPC) and GM1 (ovine brain) were purchased from Avanti Polar Lipids. Gangliosides GD1a (bovine brain), GD1b (bovine brain), and GT1b (bovine brain) and bovine serum albumin (BSA) were purchased from Sigma-Aldrich. Texas Red DHPE and cholera toxin B subunit (CTB) were purchased from ThermoFisher Scientific. The GD1a-1 antibody was obtained from the Developmental Studies Hybridoma Bank at the University of Iowa (DSHB Hybridoma Product GD1a-1). All buffer chemicals and detergents were obtained from SigmaAldrich or Fisher and were used as received. All water used to make buffers was $18 \mathrm{M} \Omega-\mathrm{cm}$ 
ultrapure $\mathrm{H}_{2} \mathrm{O}$ (PureLab Classic UV, ELGA LabWater). Three different Tris buffers were used in this work: “Tris1" (10 mM Tris, $150 \mathrm{mM} \mathrm{NaCl}, \mathrm{pH} 7.0)$, "Tris2" (10 mM Tris, $250 \mathrm{mM} \mathrm{NaCl}, \mathrm{pH}$ 7.0), and "Tris3" (10 mM Tris, $\left.150 \mathrm{mM} \mathrm{NaCl}, 2.2 \mathrm{mM} \mathrm{CaCl}_{2}, \mathrm{pH} 7.0\right)$, $\mathrm{pH}$ was adjusted dropwise with concentrated $\mathrm{HCl}$.

\section{Vesicle Preparation}

Vesicles were prepared by mixing lipids in chloroform or chloroform/methanol $(2: 1 \mathrm{v} / \mathrm{v})$ : DOPC only, DOPC/GM1 (1-5 mol\%), DOPC/GD1a (1-5 mol\%), DOPC/GD1b (1-5 mol\%), or DOPC/GT1b (1-5 mol\%). The solvent was then evaporated under vacuum overnight. Dried lipid films were rehydrated with Tris 1 buffer to give a final lipid concentration of $1 \mathrm{mg} / \mathrm{mL}$. Vesicles were made by high-speed vortexing the solution for $10 \mathrm{sec}$, bath sonicating for $10 \mathrm{~min}$, and finally extruding (Mini-Extruder, Avanti Polar Lipids) through a $50 \mathrm{~nm}$ diameter pore filter (polycarbonate track etch membranes, Avanti Polar Lipids) a total of 23 passes. Prior to experiments, vesicles were diluted in either Tris 1 buffer or Tris 3 buffer to a lipid concentration of $0.1 \mathrm{mg} / \mathrm{mL}$.

\section{QCM-D Measurements}

The QCM-D (Q-Sense E1 Explorer, Biolin Scientific) experiments were performed on AT-cut quartz crystals with a gold surface coated with $\mathrm{SiO}_{2}$ (Biolin, Q-Sense chip QSX 303). Quartz crystals were prepared by cleaning with a stream of $\mathrm{N}_{2}$ gas, 10 min UV ozone treatment (ProCleaner Plus, Bioforce Nanosciences), a 30 min soak in 2\% (w/v) sodium dodecyl sulfate (SDS) solution, rinsing with ultrapure water, drying with $\mathrm{N}_{2}$ gas, and 10 min UV ozone. After cleaning, the sensors were mounted in the flow cell and liquid was flowed over them within $5 \mathrm{~min}$. The sensor was driven at $5 \mathrm{MHz}$ and several overtones. The $3^{\text {rd }}$ overtone was used for all analyses. 
The flow cell was held at $23.0^{\circ} \mathrm{C}$. The injection sequence for SLB formation was Tris 1 , vesicles in Tris1 or Tris3, Tris1, Tris2, Tris1. The goal of the short high ionic strength Tris2 injection was to induce the rupture of intact adsorbed vesicles with osmotic stress. In binding experiments with CTB or antibodies, the injection sequence was Tris1, vesicles in Tris3, Tris1, antibody in Tris1, Tris1. The flow rate was held at $0.1 \mathrm{~mL} / \mathrm{min}$ throughout all steps.

\section{FRAP Measurements}

For FRAP experiments, $0.1 \mathrm{~mol} \%$ of the fluorophore Texas Red-DHPE was included in all vesicle mixtures. Vesicles were prepared with a $1 \mathrm{mg} / \mathrm{mL}$ lipid concentration in Tris 1 buffer and diluted $10 \times$ in Tris 3 right before adding to a glass coverslip. The glass coverslips were cleaned by soaking in $2 \%(\mathrm{v} / \mathrm{v})$ Hellmanex III at $35^{\circ} \mathrm{C}$ for $40 \mathrm{~min}$, followed by rinsing with ultrapure $\mathrm{H}_{2} \mathrm{O}$, and drying with a stream of $\mathrm{N}_{2}$ gas. The PDMS wells (6 $\mathrm{mm}$ diameter) were bonded to the coverslips by activating both surfaces with a plasma cleaner (Harrick Plasma, Ithaca, NY) using atmospheric gases at $\sim 270$ mTorr on "high" for $1.5 \mathrm{~min}$. Vesicles were added to the wells within $20 \mathrm{~min}$, and then incubated for 1 hour, followed by washing with Tris 1 to rinse away loose vesicles. After the Tris1 wash SLBs were examined with FRAP. Then the SLBs were washed with Tris1 containing $2 \mathrm{mM}$ EDTA to chelate residual $\mathrm{Ca}^{2+}$, and finally washed with Tris1 prior to additional FRAP analysis.

FRAP measurements were conducted with an inverted microscope (Eclipse Ti-E, Nikon) in epifluorescence mode with a $100 \times$ oil immersion objective $(\mathrm{NA}=1.49)$. Fluorescence was excited using an LED lamp source (Aura II, Lumencor) and a Texas Red filter set (modified TRF49909, Chroma) and captured with an air-cooled $2048 \times 2048$ pixel sCMOS camera (Orca Flash 4.0 v2, Hamamatsu). Fluorophores were bleached in a circular spot by focusing a $405 \mathrm{~nm}$ laser $(50 \mathrm{~mW})$ 
on the sample for $3 \mathrm{sec}$, and images collected with the Texas Red filter set every $1 \mathrm{sec}$ for $2 \mathrm{~min}$. FRAP analysis was performed using the Hankel transform MATLAB code created by Jönsson et al.[66] FRAP images made using the FIJI version of ImageJ.[79]

\section{Statistical Analysis}

Paired t-tests were used to compare diffusion coefficients before and after addition of EDTA. One-way ANOVAs (Dunnett test, grouped by ganglioside and compared to control DOPC-only vesicles) were used to compare QCM-D data. All statistical tests were done in Prism 8, GraphPad Software.

Supporting Information. Additional data and figures are available as supporting information. These files are available free of charge.

\section{AUTHOR INFORMATION}

\section{Corresponding Author}

*Email: njw@lehigh.edu (N.J.W)

\$These authors contributed equally.

\section{ORCID}

Nathan J. Wittenberg: 0000-0001-9196-1867

\section{Author Contributions}

L.R.J., M.E.B, A.M.B, J.L.C., and N.J.W. designed experiments; L.R.J., M.E.B, A.M.B, and J.L.C. carried out the experiments; L.R.J. M.E.B, A.M.B, J.L.C., and N.J.W. analyzed the data; L.R.J. 
and N.J.W. wrote the manuscript with input from all co-authors. M.E.B., A.M.B, and J.L.C. contributed equally.

\section{ACKNOWLEDGMENTS}

The authors acknowledge funding from Lehigh University. The anti-GD1a-1 monoclonal antibody, developed by Ronald L. Schnaar of the Johns Hopkins University School of Medicine, was obtained from the Developmental Studies Hybridoma Bank, created by the NICHD of the NIH and maintained at The University of Iowa, Department of Biology, Iowa City, IA 52242.

\section{REFERENCES}

[1] K. Vajn, B. Viljetic, I.V. Degmecic, R.L. Schnaar, M. Heffer, Differential distribution of major brain gangliosides in the adult mouse central nervous system, PLoS One, 8 (2013) e75720.

[2] R.L. Schnaar, R. Gerardy-Schahn, H. Hildebrandt, Sialic acids in the brain: gangliosides and polysialic acid in nervous system development, stability, disease, and regeneration, Physiol Rev, 94 (2014) 461-518.

[3] G. Tettamanti, F. Bonali, S. Marchesini, V. Zambotti, A new procedure for the extraction, purification and fractionation of brain gangliosides, Biochim Biophys Acta, 296 (1973) 160-170.

[4] S. Sonnino, L. Mauri, V. Chigorno, A. Prinetti, Gangliosides as components of lipid membrane domains, Glycobiology, 17 (2007) 1R-13R.

[5] M.M. Lozano, Z. Liu, E. Sunnick, A. Janshoff, K. Kumar, S.G. Boxer, Colocalization of the ganglioside G(M1) and cholesterol detected by secondary ion mass spectrometry, J Am Chem Soc, 135 (2013) 5620-5630.

[6] J. Shi, T. Yang, S. Kataoka, Y. Zhang, A.J. Diaz, P.S. Cremer, GM1 clustering inhibits cholera toxin binding in supported phospholipid membranes, J Am Chem Soc, 129 (2007) 5954-5961.

[7] L.J. Yang, C.B. Zeller, N.L. Shaper, M. Kiso, A. Hasegawa, R.E. Shapiro, R.L. Schnaar, Gangliosides are neuronal ligands for myelin-associated glycoprotein, Proc Natl Acad Sci U S A, 93 (1996) 814-818.

[8] R.A. Hughes, D.R. Cornblath, Guillain-Barre syndrome, Lancet, 366 (2005) 1653-1666.

[9] M.S. Ravindran, L.B. Tanner, M.R. Wenk, Sialic acid linkage in glycosphingolipids is a molecular correlate for trafficking and delivery of extracellular cargo, Traffic, 14 (2013) 11821191.

[10] G.M. Kuziemko, M. Stroh, R.C. Stevens, Cholera toxin binding affinity and specificity for gangliosides determined by surface plasmon resonance, Biochemistry, 35 (1996) 6375-6384.

[11] W.E. Minke, C. Roach, W.G. Hol, C.L. Verlinde, Structure-based exploration of the ganglioside GM1 binding sites of Escherichia coli heat-labile enterotoxin and cholera toxin for the discovery of receptor antagonists, Biochemistry, 38 (1999) 5684-5692. 
[12] C. Montecucco, G. Schiavo, Structure and function of tetanus and botulinum neurotoxins, Q Rev Biophys, 28 (1995) 423-472.

[13] K. Yanagisawa, A. Odaka, N. Suzuki, Y. Ihara, GM1 ganglioside-bound amyloid beta-protein (A beta): a possible form of preamyloid in Alzheimer's disease, Nat Med, 1 (1995) 1062-1066.

[14] R. Gaspar, J. Pallbo, U. Weininger, S. Linse, E. Sparr, Ganglioside lipids accelerate alphasynuclein amyloid formation, Biochim Biophys Acta Proteins Proteom, (2018).

[15] E.T. Castellana, P.S. Cremer, Solid supported lipid bilayers: From biophysical studies to sensor design, Surf Sci Rep, 61 (2006) 429-444.

[16] R.P. Richter, R. Berat, A.R. Brisson, Formation of solid-supported lipid bilayers: an integrated view, Langmuir, 22 (2006) 3497-3505.

[17] F. Mazur, M. Bally, B. Stadler, R. Chandrawati, Liposomes and lipid bilayers in biosensors, Adv Colloid Interface Sci, 249 (2017) 88-99.

[18] A.K. Singh, S.H. Harrison, J.S. Schoeniger, Gangliosides as receptors for biological toxins: development of sensitive fluoroimmunoassays using ganglioside-bearing liposomes, Anal Chem, 72 (2000) 6019-6024.

[19] J.M. Moran-Mirabal, J.B. Edel, G.D. Meyer, D. Throckmorton, A.K. Singh, H.G. Craighead, Micrometer-sized supported lipid bilayer arrays for bacterial toxin binding studies through total internal reflection fluorescence microscopy, Biophys J, 89 (2005) 296-305.

[20] N.J. Wittenberg, T.W. Johnson, S.H. Oh, High-density arrays of submicron spherical supported lipid bilayers, Anal Chem, 84 (2012) 8207-8213.

[21] A.M. Kabbani, C.V. Kelly, Nanoscale Membrane Budding Induced by CTxB and Detected via Polarized Localization Microscopy, Biophys J, 113 (2017) 1795-1806.

[22] N. Parveen, I. Rimkute, S. Block, G.E. Rydell, D. Midtvedt, G. Larson, V.P. Hytonen, V.P. Zhdanov, A. Lundgren, F. Hook, Membrane Deformation Induces Clustering of Norovirus Bound to Glycosphingolipids in a Supported Cell-Membrane Mimic, J Phys Chem Lett, 9 (2018) 22782284.

[23] M.M. Baksh, A.K. Kussrow, M. Mileni, M.G. Finn, D.J. Bornhop, Label-free quantification of membrane-ligand interactions using backscattering interferometry, Nat Biotechnol, 29 (2011) 357-360.

[24] M.M. Baksh, M. Jaros, J.T. Groves, Detection of molecular interactions at membrane surfaces through colloid phase transitions, Nature, 427 (2004) 139-141.

[25] S.H. Lee, N.C. Lindquist, N.J. Wittenberg, L.R. Jordan, S.H. Oh, Real-time full-spectral imaging and affinity measurements from 50 microfluidic channels using nanohole surface plasmon resonance, Lab Chip, 12 (2012) 3882-3890.

[26] X. Xu, A. Denic, L.R. Jordan, N.J. Wittenberg, A.E. Warrington, B. Wootla, L.M. Papke, L.J. Zoecklein, D. Yoo, J. Shaver, S.H. Oh, L.R. Pease, M. Rodriguez, A natural human IgM that binds to gangliosides is therapeutic in murine models of amyotrophic lateral sclerosis, Dis Model Mech, 8 (2015) 831-842.

[27] H.J. Wu, J. Henzie, W.C. Lin, C. Rhodes, Z. Li, E. Sartorel, J. Thorner, P. Yang, J.T. Groves, Membrane-protein binding measured with solution-phase plasmonic nanocube sensors, Nat Methods, 9 (2012) 1189-1191.

[28] W.B. Turnbull, B.L. Precious, S.W. Homans, Dissecting the cholera toxin-ganglioside GM1 interaction by isothermal titration calorimetry, J Am Chem Soc, 126 (2004) 1047-1054.

[29] A. Janshoff, C. Steinem, M. Sieber, A. el Baya, M.A. Schmidt, H.J. Galla, Quartz crystal microbalance investigation of the interaction of bacterial toxins with ganglioside containing solid supported membranes, Eur Biophys J, 26 (1997) 261-270. 
[30] K.C. Weng, J.L. Kanter, W.H. Robinson, C.W. Frank, Fluid supported lipid bilayers containing monosialoganglioside GM1: a QCM-D and FRAP study, Colloids Surf B Biointerfaces, 50 (2006) 76-84.

[31] A.A. Brian, H.M. McConnell, Allogeneic stimulation of cytotoxic T cells by supported planar membranes, Proc Natl Acad Sci U S A, 81 (1984) 6159-6163.

[32] L.K. Tamm, H.M. McConnell, Supported phospholipid bilayers, Biophys J, 47 (1985) 105113.

[33] S.R. Tabaei, J.H. Choi, G. Haw Zan, V.P. Zhdanov, N.J. Cho, Solvent-assisted lipid bilayer formation on silicon dioxide and gold, Langmuir, 30 (2014) 10363-10373.

[34] M.D. Mager, N.A. Melosh, Lipid bilayer deposition and patterning via air bubble collapse, Langmuir, 23 (2007) 9369-9377.

[35] B. Seantier, C. Breffa, O. Felix, G. Decher, Dissipation-enhanced quartz crystal microbalance studies on the experimental parameters controlling the formation of supported lipid bilayers, J Phys Chem B, 109 (2005) 21755-21765.

[36] S. Jo, T. Kim, V.G. Iyer, W. Im, CHARMM-GUI: a web-based graphical user interface for CHARMM, J Comput Chem, 29 (2008) 1859-1865.

[37] S. Groux-Degroote, Y. Guerardel, P. Delannoy, Gangliosides: Structures, Biosynthesis, Analysis, and Roles in Cancer, Chembiochem, 18 (2017) 1146-1154.

[38] R.Y. Patel, P.V. Balaji, Characterization of symmetric and asymmetric lipid bilayers composed of varying concentrations of ganglioside GM1 and DPPC, J Phys Chem B, 112 (2008) 3346-3356.

[39] C. Yuan, L.J. Johnston, Distribution of ganglioside GM1 in L-alphadipalmitoylphosphatidylcholine/cholesterol monolayers: a model for lipid rafts, Biophys J, 79 (2000) 2768-2781.

[40] I. Reviakine, D. Johannsmann, R.P. Richter, Hearing what you cannot see and visualizing what you hear: interpreting quartz crystal microbalance data from solvated interfaces, Anal Chem, 83 (2011) 8838-8848.

[41] C.A. Keller, B. Kasemo, Surface specific kinetics of lipid vesicle adsorption measured with a quartz crystal microbalance, Biophys J, 75 (1998) 1397-1402.

[42] R. Richter, A. Mukhopadhyay, A. Brisson, Pathways of lipid vesicle deposition on solid surfaces: a combined QCM-D and AFM study, Biophys J, 85 (2003) 3035-3047.

[43] R.P. Richter, A.R. Brisson, Following the formation of supported lipid bilayers on mica: a study combining AFM, QCM-D, and ellipsometry, Biophys J, 88 (2005) 3422-3433.

[44] C.A. Keller, K. Glasmastar, V.P. Zhdanov, B. Kasemo, Formation of supported membranes from vesicles, Phys Rev Lett, 84 (2000) 5443-5446.

[45] P.S. Cremer, S.G. Boxer, Formation and spreading of lipid bilayers on planar glass supports, Journal of Physical Chemistry B, 103 (1999) 2554-2559.

[46] E. Reimhult, F. Hook, B. Kasemo, Temperature dependence of formation of a supported phospholipid bilayer from vesicles on SiO2, Phys Rev E Stat Nonlin Soft Matter Phys, 66 (2002) 051905.

[47] E. Reimhult, F. Hook, B. Kasemo, Vesicle adsorption on $\mathrm{SiO} 2$ and TiO2: Dependence on vesicle size, J Chem Phys, 117 (2002) 7401-7404.

[48] E. Reimhult, F. Hook, B. Kasemo, Intact vesicle adsorption and supported biomembrane formation from vesicles in solution: Influence of surface chemistry, vesicle size, temperature, and osmotic pressure, Langmuir, 19 (2003) 1681-1691. 
[49] C.M. Bailey, A. Tripathi, A. Shukla, Effects of Flow and Bulk Vesicle Concentration on Supported Lipid Bilayer Formation, Langmuir, 33 (2017) 11986-11997.

[50] T.H. Anderson, Y. Min, K.L. Weirich, H. Zeng, D. Fygenson, J.N. Israelachvili, Formation of supported bilayers on silica substrates, Langmuir, 25 (2009) 6997-7005.

[51] N. Hain, M. Gallego, I. Reviakine, Unraveling supported lipid bilayer formation kinetics: osmotic effects, Langmuir, 29 (2013) 2282-2288.

[52] J.A. Jackman, J.H. Choi, V.P. Zhdanov, N.J. Cho, Influence of osmotic pressure on adhesion of lipid vesicles to solid supports, Langmuir, 29 (2013) 11375-11384.

[53] N.J. Cho, J.A. Jackman, M. Liu, C.W. Frank, pH-driven assembly of various supported lipid platforms: a comparative study on silicon oxide and titanium oxide, Langmuir, 27 (2011) 37393748.

[54] M. Dacic, J.A. Jackman, S. Yorulmaz, V.P. Zhdanov, B. Kasemo, N.J. Cho, Influence of Divalent Cations on Deformation and Rupture of Adsorbed Lipid Vesicles, Langmuir, 32 (2016) 6486-6495.

[55] N.J. Cho, C.W. Frank, B. Kasemo, F. Hook, Quartz crystal microbalance with dissipation monitoring of supported lipid bilayers on various substrates, Nat Protoc, 5 (2010) 1096-1106.

[56] T.J. McIntosh, S.A. Simon, Hydration force and bilayer deformation: a reevaluation, Biochemistry, 25 (1986) 4058-4066.

[57] U. Seifert, R. Lipowsky, Adhesion of vesicles, Phys Rev A, 42 (1990) 4768-4771.

[58] V.P. Zhdanov, B. Kasemo, Comments on rupture of absorbed vesicles, Langmuir, 17 (2001) 3518-3521.

[59] Y. Hirabayashi, A. Hyogo, T. Nakao, K. Tsuchiya, Y. Suzuki, M. Matsumoto, K. Kon, S. Ando, Isolation and characterization of extremely minor gangliosides, GM1b and GD1 alpha, in adult bovine brains as developmentally regulated antigens, J Biol Chem, 265 (1990) 8144-8151.

[60] S.L. Frey, E.Y. Chi, C. Arratia, J. Majewski, K. Kjaer, K.Y. Lee, Condensing and fluidizing effects of ganglioside GM1 on phospholipid films, Biophys J, 94 (2008) 3047-3064.

[61] S.L. Frey, K.Y. Lee, Number of sialic acid residues in ganglioside headgroup affects interactions with neighboring lipids, Biophys J, 105 (2013) 1421-1431.

[62] B. Seantier, B. Kasemo, Influence of mono- and divalent ions on the formation of supported phospholipid bilayers via vesicle adsorption, Langmuir, 25 (2009) 5767-5772.

[63] F.F. Rossetti, M. Bally, R. Michel, M. Textor, I. Reviakine, Interactions between titanium dioxide and phosphatidyl serine-containing liposomes: formation and patterning of supported phospholipid bilayers on the surface of a medically relevant material, Langmuir, 21 (2005) 64436450.

[64] S.R. Tabaei, F. Guo, F.U. Rutaganira, S. Vafaei, I. Choong, K.M. Shokat, J.S. Glenn, N.J. Cho, Multistep Compositional Remodeling of Supported Lipid Membranes by Interfacially Active Phosphatidylinositol Kinases, Anal Chem, 88 (2016) 5042-5045.

[65] S.R. Tabaei, J.A. Jackman, S.O. Kim, B. Liedberg, W. Knoll, A.N. Parikh, N.J. Cho, Formation of cholesterol-rich supported membranes using solvent-assisted lipid self-assembly, Langmuir, 30 (2014) 13345-13352.

[66] P. Jonsson, M.P. Jonsson, J.O. Tegenfeldt, F. Hook, A method improving the accuracy of fluorescence recovery after photobleaching analysis, Biophys J, 95 (2008) 5334-5348.

[67] L.K. Tamm, Lateral diffusion and fluorescence microscope studies on a monoclonal antibody specifically bound to supported phospholipid bilayers, Biochemistry, 27 (1988) 1450-1457. 
[68] L.B. Sagle, L.K. Ruvuna, J.M. Bingham, C. Liu, P.S. Cremer, R.P. Van Duyne, Single plasmonic nanoparticle tracking studies of solid supported bilayers with ganglioside lipids, J Am Chem Soc, 134 (2012) 15832-15839.

[69] F.J. Sharom, C.W. Grant, A model for ganglioside behaviour in cell membranes, Biochim Biophys Acta, 507 (1978) 280-293.

[70] L. Svennerholm, The Gangliosides, J Lipid Res, 5 (1964) 145-155.

[71] L. Silva, R.F. de Almeida, A. Fedorov, A.P. Matos, M. Prieto, Ceramide-platform formation and -induced biophysical changes in a fluid phospholipid membrane, Mol Membr Biol, 23 (2006) 137-148.

[72] A. Melcrova, S. Pokorna, S. Pullanchery, M. Kohagen, P. Jurkiewicz, M. Hof, P. Jungwirth, P.S. Cremer, L. Cwiklik, The complex nature of calcium cation interactions with phospholipid bilayers, Sci Rep, 6 (2016) 38035.

[73] R. McDaniel, S. McLaughlin, The interaction of calcium with gangliosides in bilayer membranes, Biochim Biophys Acta, 819 (1985) 153-160.

[74] R.A. Bockmann, H. Grubmuller, Multistep binding of divalent cations to phospholipid bilayers: a molecular dynamics study, Angew Chem Int Ed Engl, 43 (2004) 1021-1024.

[75] H. Binder, O. Zschornig, The effect of metal cations on the phase behavior and hydration characteristics of phospholipid membranes, Chem Phys Lipids, 115 (2002) 39-61.

[76] L. Svennerholm, K. Bostrom, P. Fredman, B. Jungbjer, A. Lekman, J.E. Mansson, B.M. Rynmark, Gangliosides and allied glycosphingolipids in human peripheral nerve and spinal cord, Biochim Biophys Acta, 1214 (1994) 115-123.

[77] Y. Gong, Y. Tagawa, M.P. Lunn, W. Laroy, M. Heffer-Lauc, C.Y. Li, J.W. Griffin, R.L. Schnaar, K.A. Sheikh, Localization of major gangliosides in the PNS: implications for immune neuropathies, Brain, 125 (2002) 2491-2506.

[78] P.H. Lopez, G. Zhang, M.A. Bianchet, R.L. Schnaar, K.A. Sheikh, Structural requirements of anti-GD1a antibodies determine their target specificity, Brain, 131 (2008) 1926-1939.

[79] J. Schindelin, I. Arganda-Carreras, E. Frise, V. Kaynig, M. Longair, T. Pietzsch, S. Preibisch, C. Rueden, S. Saalfeld, B. Schmid, J.Y. Tinevez, D.J. White, V. Hartenstein, K. Eliceiri, P. Tomancak, A. Cardona, Fiji: an open-source platform for biological-image analysis, Nat Methods, 9 (2012) 676-682. 


\section{Supplementary Material}

\section{Influence of Brain Gangliosides on the Formation and Properties of Supported Lipid Bilayers}

Luke R. Jordan, ${ }^{a}$ Megan E. Blauch ${ }^{a, b}$ Ashley M. Baxter, ${ }^{a, b}$ Jennie L. Cawley, ${ }^{a, b}$ and Nathan J. Wittenberg $^{a *}$

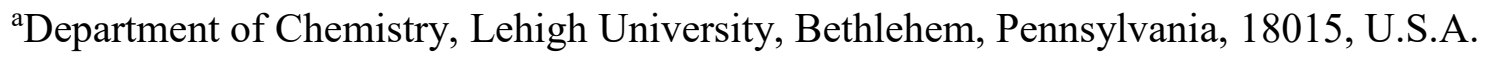

${ }^{\mathrm{b}}$ These authors contributed equally.

*Corresponding author. Email address: njw@lehigh.edu 

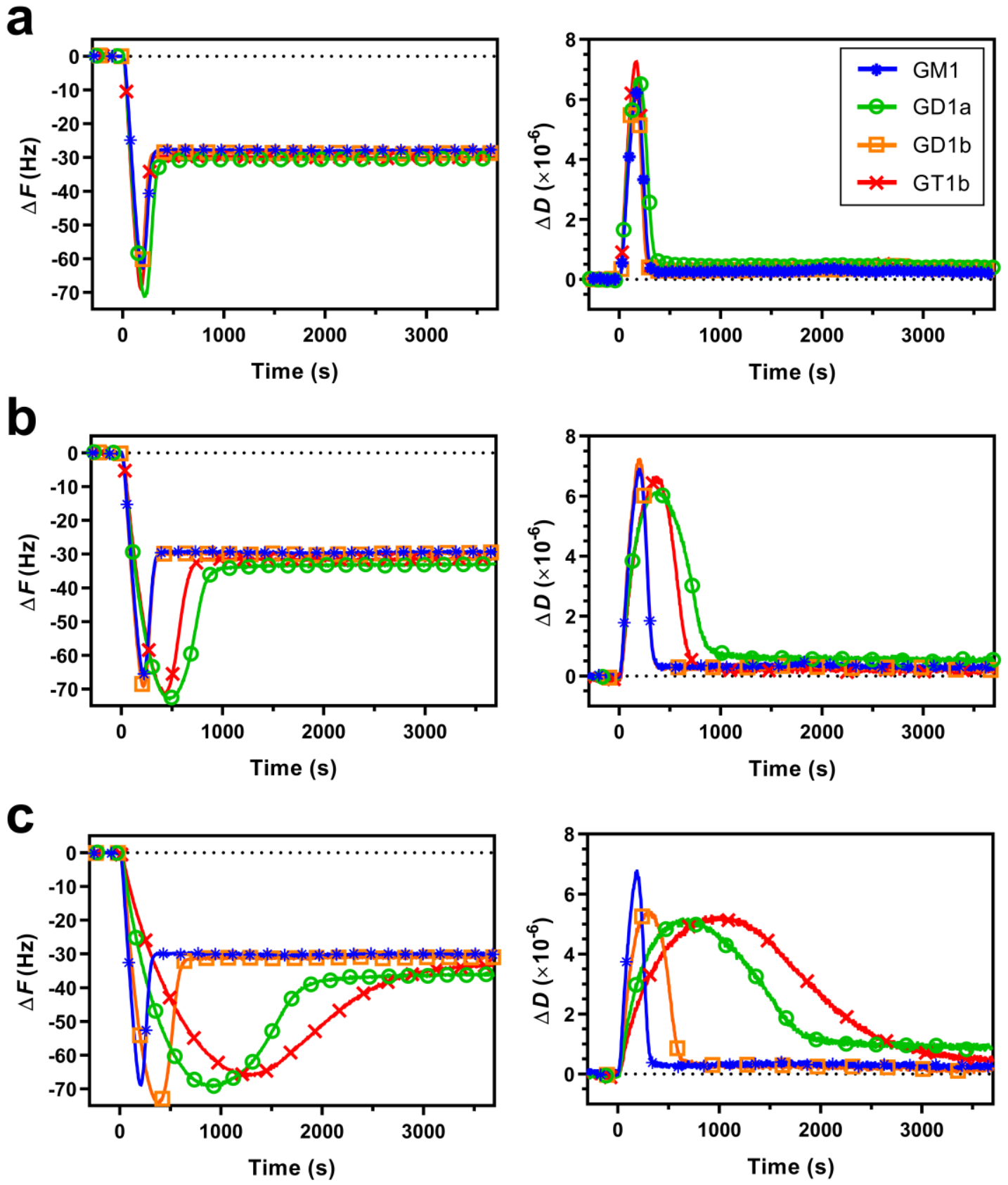

Figure S1. Formation of SLBs containing $2-4 \%$ gangliosides in the absence of calcium. Frequency (left) and dissipation (right) shifts for adsorption and rupture of DOPC vesicles with (b) $2 \%$, (c) $3 \%$, and (d) $4 \%$ gangliosides (GM1, GD1a, GD1b, and GT1b). 

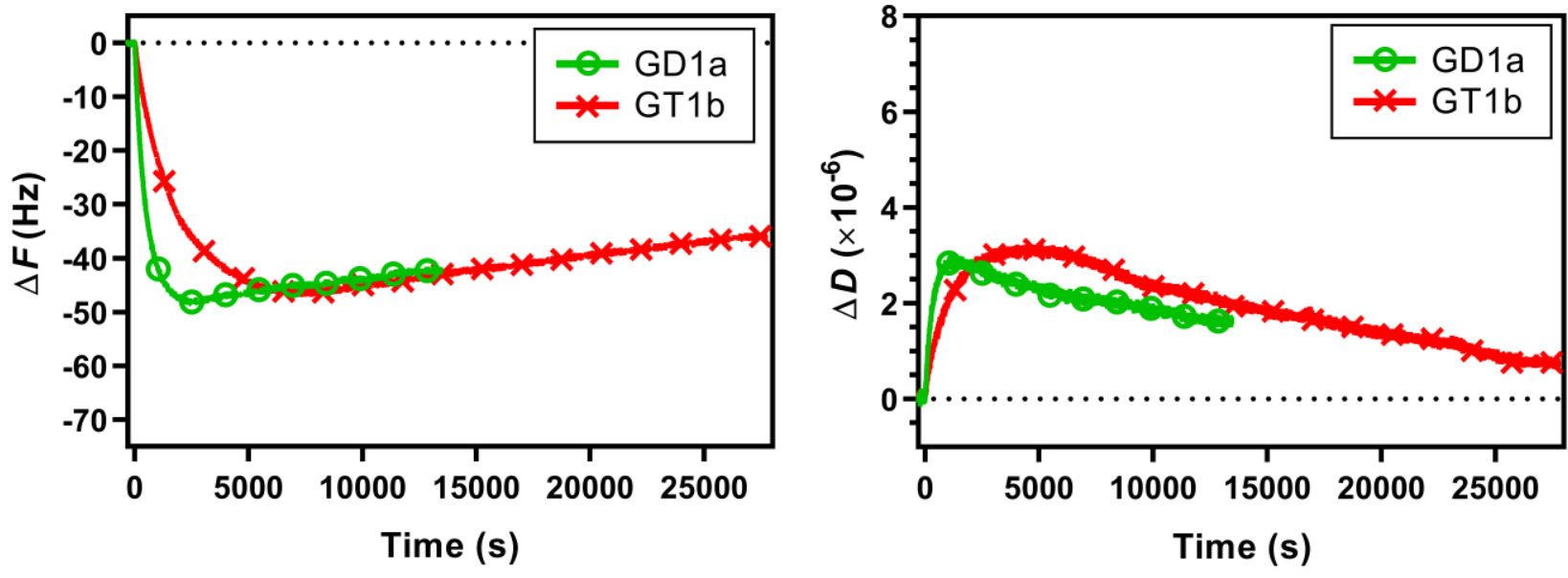

Figure S2: Time course of adsorption and rupture of vesicles with $5 \%$ GD1a and $5 \%$ GT1b in Tris buffer lacking $\mathrm{Ca}^{2+}$. Panels show QCM-D frequency (left) and dissipation (right) shifts. Figure $2 \mathrm{c}$ in the main manuscript shows only the first hour $(3600 \mathrm{~s})$ of these curves.

Table S1. Lipid diffusion coefficients determined from FRAP measurements.

\begin{tabular}{|c|c|c|c|c|c|c|}
\hline Sample & $\begin{array}{c}D \text { before } \\
\text { EDTA }\left(\mu \mathrm{m}^{2} / \mathrm{s}\right) \\
\text { Mean } \pm \text { S.D. }\end{array}$ & $\begin{array}{c}\text { N before } \\
\text { EDTA }\end{array}$ & $\begin{array}{c}D \text { after EDTA } \\
\left(\mu \mathrm{m}^{2} / \mathrm{s}\right) \\
\text { Mean } \pm \text { S.D. }\end{array}$ & $\mathbf{N}$ after EDTA & P value ${ }^{a}$ & Significant? $^{b}$ \\
\hline $100 \%$ DOPC & $2.30 \pm 0.22$ & 15 & $2.75 \pm 0.26$ & 15 & $<0.0001$ & Yes \\
\hline $1 \%$ GM1 & $2.12 \pm 0.18$ & 15 & $2.47 \pm 0.30$ & 15 & 0.0007 & Yes \\
\hline $3 \%$ GM1 & $1.96 \pm 0.10$ & 14 & $2.40 \pm 0.13$ & 14 & $<0.0001$ & Yes \\
\hline $5 \%$ GM1 & $1.63 \pm 0.12$ & 15 & $1.74 \pm 0.09$ & 15 & 0.0005 & Yes \\
\hline $1 \%$ GD1a & $2.30 \pm 0.14$ & 14 & $2.69 \pm 0.09$ & 14 & $<0.0001$ & Yes \\
\hline $3 \%$ GD1a & $2.05 \pm 0.14$ & 15 & $2.24 \pm 0.12$ & 15 & 0.0023 & Yes \\
\hline $5 \%$ GD1a & $2.05 \pm 0.22$ & 14 & $1.85 \pm 0.48$ & 14 & 0.0907 & No \\
\hline $1 \%$ GD1b & $2.26 \pm 0.16$ & 15 & $2.56 \pm 0.11$ & 15 & $<0.0001$ & Yes \\
\hline $3 \%$ GD1b & $1.91 \pm 0.10$ & 14 & $2.22 \pm 0.10$ & 14 & $<0.0001$ & Yes \\
\hline $5 \%$ GD1b & $1.67 \pm 0.15$ & 14 & $1.93 \pm 0.14$ & 14 & $<0.0001$ & Yes \\
\hline $1 \%$ GT1b & $2.72 \pm 0.18$ & 14 & $2.64 \pm 0.16$ & 14 & 0.3084 & No \\
\hline $3 \%$ GT1b & $2.22 \pm 0.13$ & 15 & $2.40 \pm 0.17$ & 15 & 0.0074 & Yes \\
\hline $5 \%$ GT1b & $1.78 \pm 0.09$ & 15 & $2.05 \pm 0.19$ & 15 & 0.0002 & Yes \\
\hline
\end{tabular}

${ }^{\text {a }} P$ value for paired t-tests comparing diffusion coefficients for SLBs before and after EDTA addition. ${ }^{\text {bS }}$ Samples were identified as significantly different if $P<0.05$. 


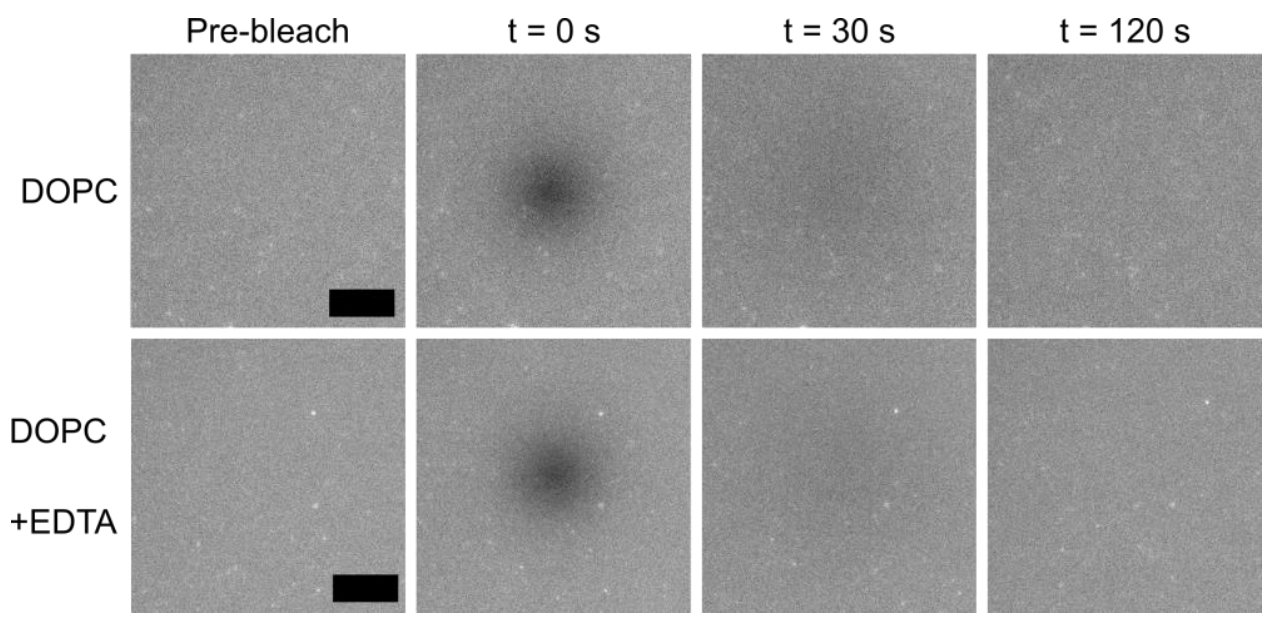

Figure S3. Fluorescence recovery after photobleaching (FRAP) images of DOPC supported lipid bilayers before and after $\mathrm{Ca}^{2+}$ chelation with EDTA. Scale bar $=10 \mu \mathrm{m}$. 


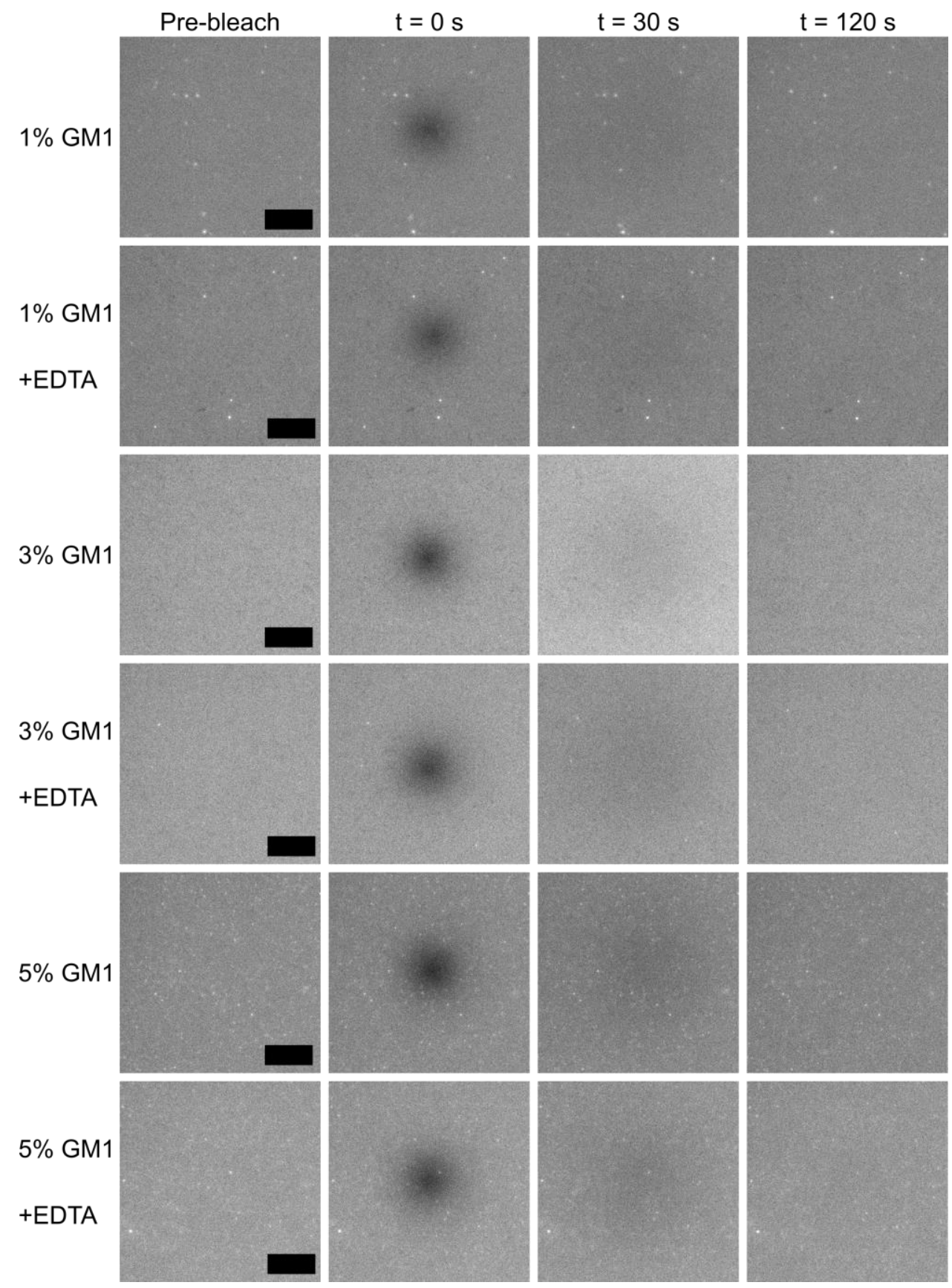

Figure S4. Fluorescence recovery after photobleaching (FRAP) images of DOPC supported lipid bilayers containing 1, 3, or 5\% GM1 before and after $\mathrm{Ca}^{2+}$ chelation with EDTA. Scale bar $=10$ $\mu \mathrm{m}$. 


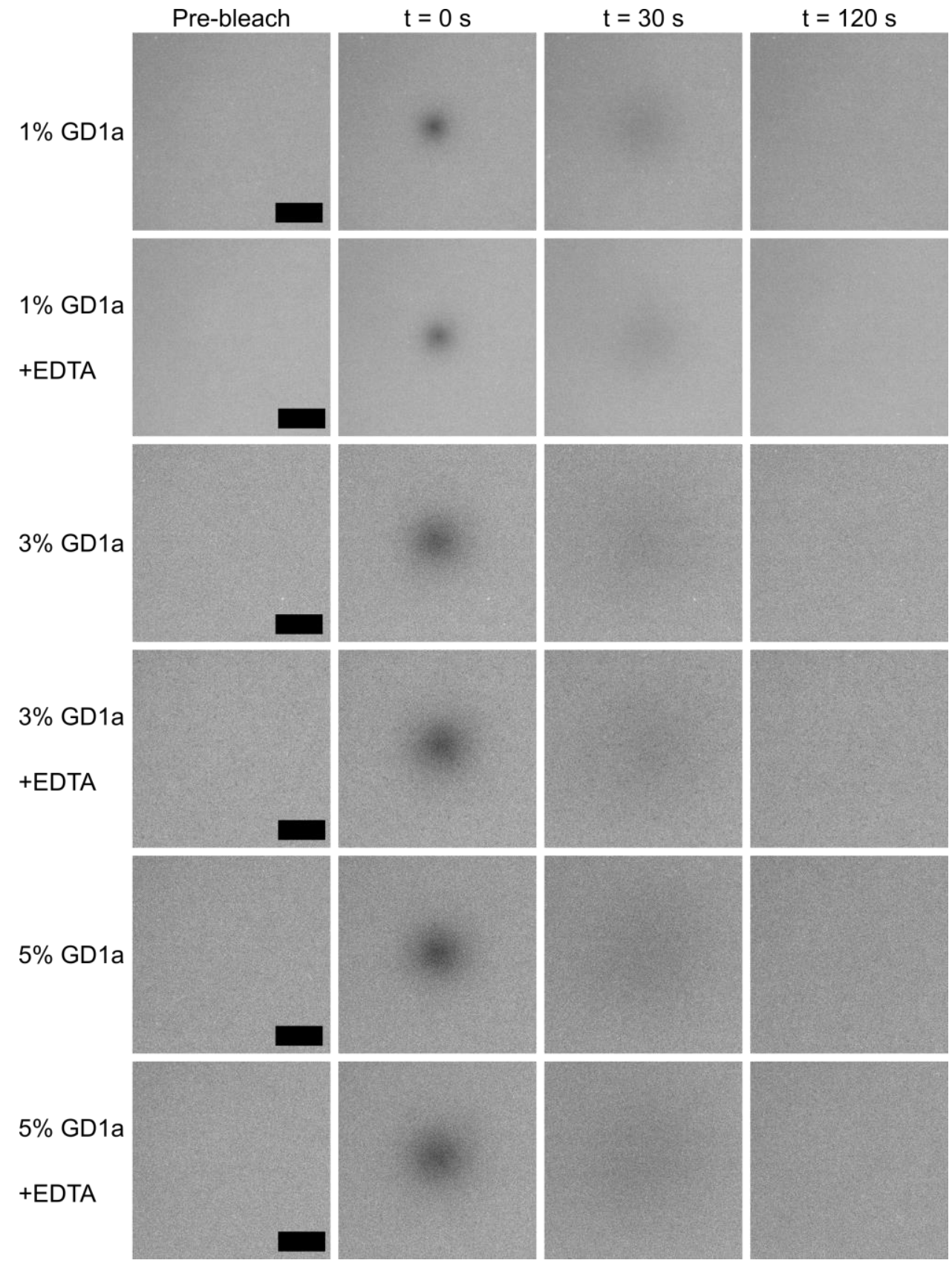

Figure S5. Fluorescence recovery after photobleaching (FRAP) images of DOPC supported lipid bilayers containing 1,3 , or $5 \%$ GD1a before and after $\mathrm{Ca}^{2+}$ chelation with EDTA. Scale bar $=10$ $\mu \mathrm{m}$. 


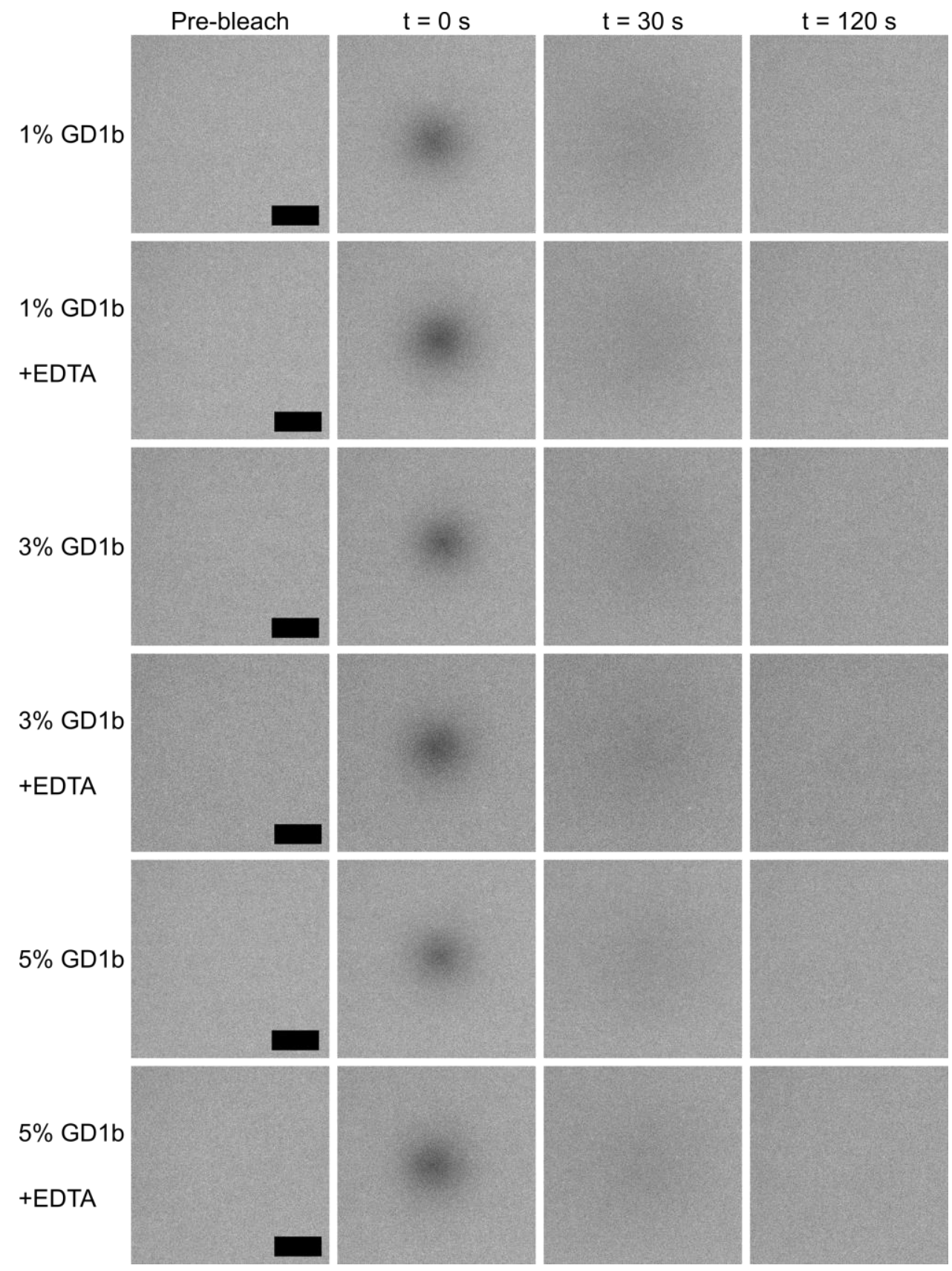

Figure S6. Fluorescence recovery after photobleaching (FRAP) images of DOPC supported lipid bilayers containing 1, 3, or 5\% GD1b before and after $\mathrm{Ca}^{2+}$ chelation with EDTA. Scale bar $=10$ $\mu \mathrm{m}$. 


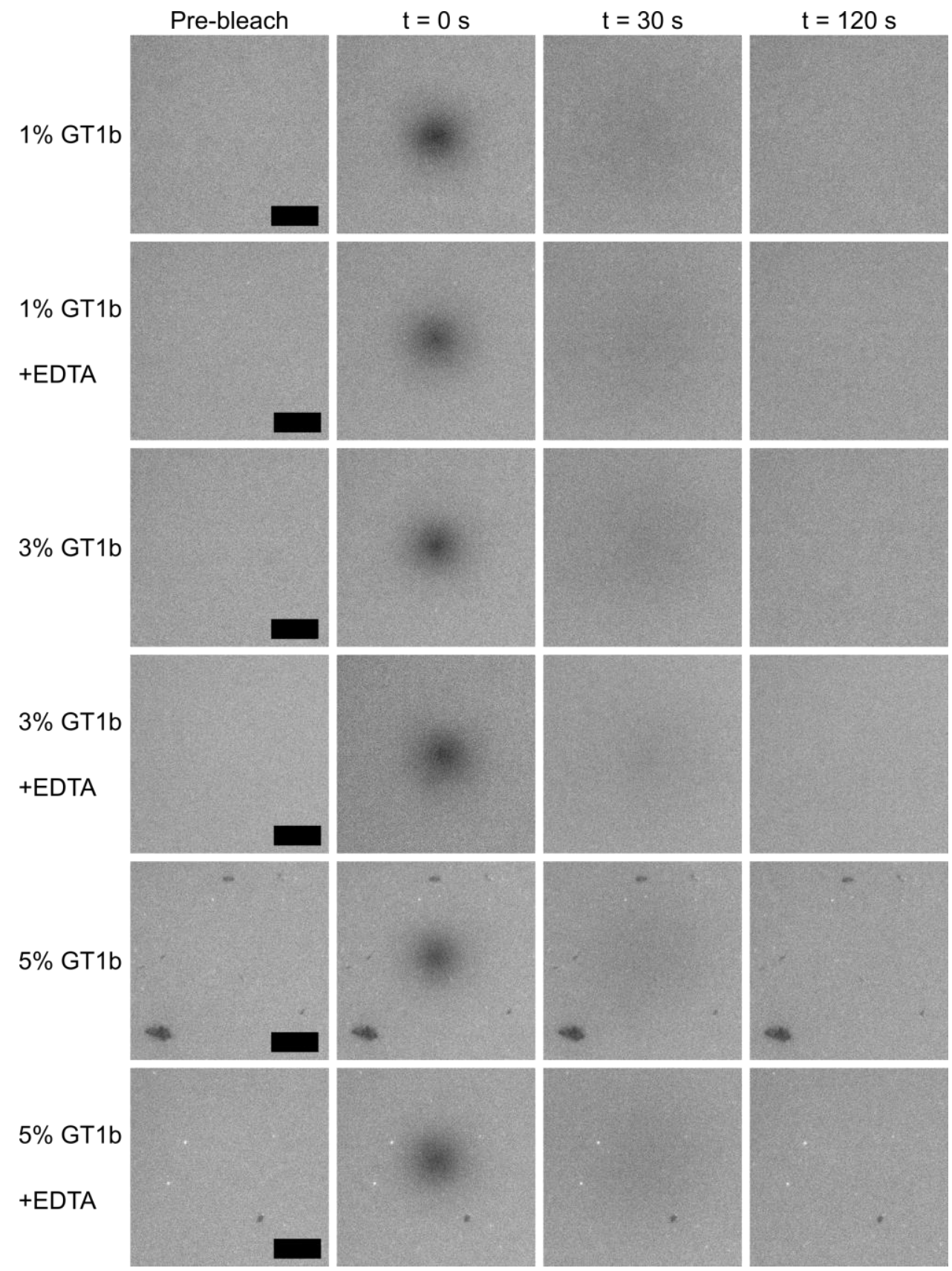

Figure S7. Fluorescence recovery after photobleaching (FRAP) images of DOPC supported lipid bilayers containing 1,3, or 5\% GT1b before and after $\mathrm{Ca}^{2+}$ chelation with EDTA. Scale bar $=10$ $\mu \mathrm{m}$. 


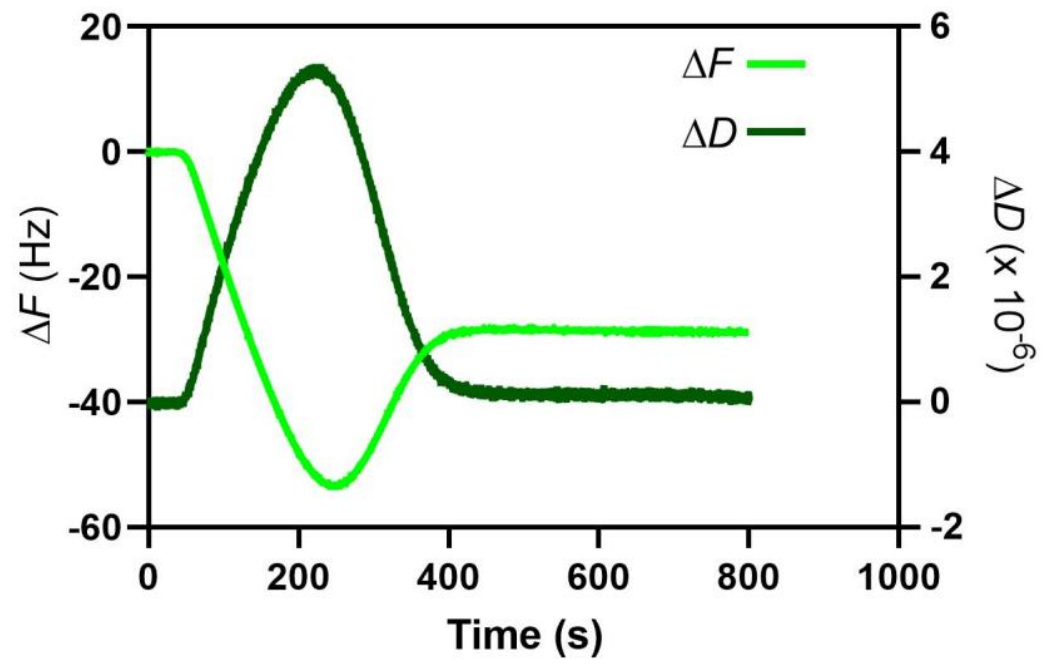

Figure S8. Formation of a SLB containing 1\% GD1a in the presence of $2 \mathrm{mM} \mathrm{Ca}^{2+}$ monitored with QCM-D. The light green curve represents frequency shift $(\Delta F)$ and is plotted against the left $y$-axis. The dark green curve represents dissipation shift $(\Delta D)$ and is plotted against the right yaxis.

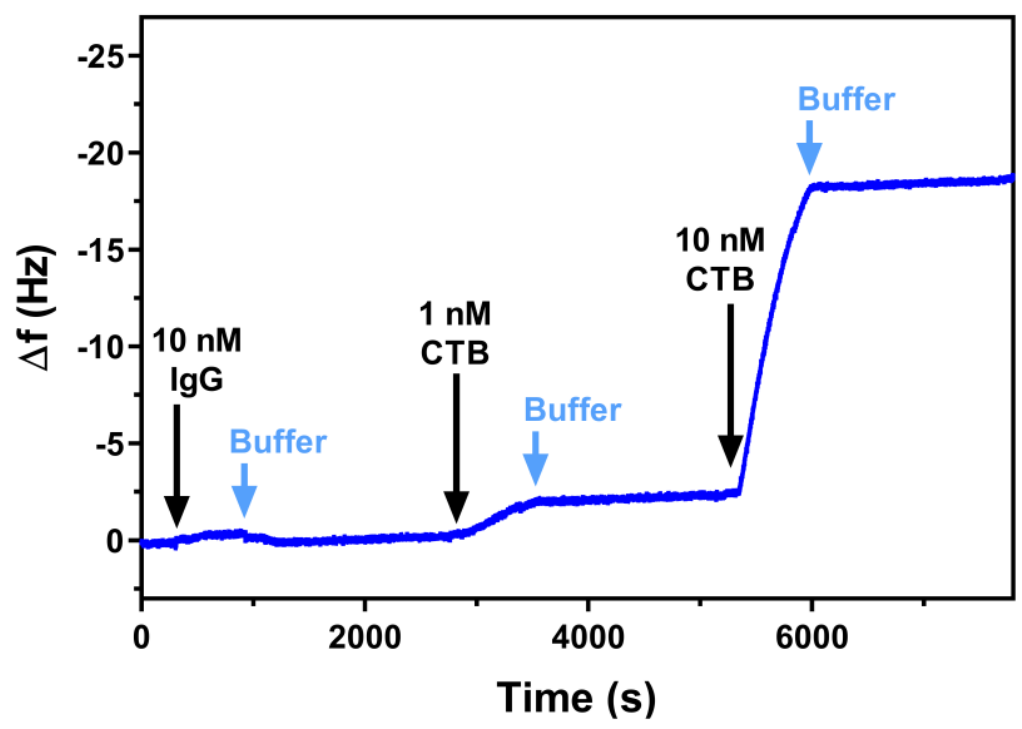

Figure S9. Cholera toxin B subunit (CTB) binding to SLB with 5\% GM1. The GD1a-1 antibody was first injected over the SLB, and it did not appreciably bind to the SLB. Subsequent injections of 1 and $10 \mathrm{nM}$ of CTB show significant binding and negligible dissociation, indicating a very high affinity interaction. 


\section{Curve fitting for antibody binding assays}

Curves were fit to the QCM-D data of binding between anti-GD1a IgG to GD1a-rich SLBs in the following manner. First, an exponential curve was fit to the dissociation phase data, using the following equation ${ }^{1}$ :

$R_{D}(t)=R_{o} e^{-k_{d} t} \quad$ Eq. $\mathbf{S 1}$

where $R_{D}(t)$ is the sensor response as a function of time $(t), R_{o}$ is the magnitude of the sensor response when dissociation begins, and $k_{\mathrm{d}}$ is the dissociation rate constant.

Next, an exponential curve was fit to the association phase data using Eq. S2 ${ }^{1}$ by inserting the $k_{\mathrm{d}}$ value calculated in Eq. S1:

$R_{A}(t)=\frac{C k_{a} R_{\max }\left[1-e^{-\left(C k_{a}+k_{d}\right) t}\right]}{C k_{a}+k_{d}} \quad$ Eq. $\mathbf{S 2}$

where $R_{A}(t)$ is the sensor response as a function of time, $R_{\max }$ is the maximum sensor response for a given antibody concentration, $C$ is the bulk concentration of antibodies in solution, and $k_{\mathrm{a}}$ is the antibody association rate constant.

\section{Supporting Information References}

1. O'Shannessy, D. J.; Brigham-Burke, M.; Soneson, K. K.; Hensley, P.; Brooks, I., Determination of rate and equilibrium binding constants for macromolecular interactions using surface plasmon resonance: use of nonlinear least squares analysis methods. Anal Biochem 1993, 212 (2), 457-68. 
